\title{
Das Archiv des Instituts für Radiumforschung
}

\author{
Von \\ Stefan Sienell und Christine Ottner
}

(Vorgelegt in der Sitzung der math.-nat. Klasse am 14. Oktober 2004 durch das w. M. Hermann Hunger)

Am 7. Dezember 1934 informierte Stefan Meyer in seiner Eigenschaft als Sekretär der internationalen Radium-StandardKommission Auguste Piccard, Brüssel, über dessen Kooptation und erbat eine Nachricht, ob Piccard seiner Wahl zustimme. Als Meyer zwei Wochen später noch keine Antwort aus Brüssel hatte, urgierte er bei Piccard, der jedoch seinerseits bereits am 17. Dezember an Meyer geschrieben und die Annahme seine Wahl erklärt hatte. Auf Meyers zweites Schreiben antwortete er am 29. Dezember per Einschreiben: „Ich sende diesen Brief eingeschrieben, damit er nicht den ersten in einer Autographensammlung wiederfindet. “1 Tatsächlich haben beide Briefe Piccards ihren Empfänger erreicht, und auch die Durchschläge der Briefe Meyers liegen in dessen Nachlaß. Sie sind nicht in einer Autographensammlung verloren gegangen, sondern nun der allgemeinen wissenschaftsgeschichtlichen Forschung zugänglich. Mit dem Archivbehelf ist der Bestand „Institut für Radiumforschung “ im Archiv der Österreichischen Akademie der

\footnotetext{
${ }^{1}$ Archiv der Österreichischen Akademie der Wissenschaften (AÖAW), Institut für Radiumforschung, K. 18.
} 
Wissenschaften (AÖAW) nach den Grundsätzen der archivischen Verzeichnung ${ }^{2}$ erschlossen. $^{3}$

\section{Kursorischer Abriß der Geschichte des Instituts}

Das Institut für Radiumforschung (,Radium-Institut“) wurde in den Jahren 1909/10 aus den Mitteln der Stiftung von Dr. Karl Kupelwieser ${ }^{4}$ errichtet und am 28. Oktober 1910 eröffnet. Am 13. Januar 1955 erfolgte die Umbenennung in „Institut für Radiumforschung und Kernphysik " und schließlich am 7. November 1986 die Umwandlung in das „Institut für Mittelenergiephysik“. Seit der Gründung stand Stefan Meyer dem Institut vor, mußte jedoch 1938 die Leitung zurücklegen. Ihm folgte sein langjähriger Assistent Gustav Ortner nach, der seinerseits die Geschicke des Instituts bis Kriegsende in Händen hielt. ${ }^{5}$ Mit Dekret vom 29. Dezember 1947 wurde Berta Karlik, die bis dahin unter dem zurückgekehrten Vorstand Meyer provisorische Leiterin war, offiziell als Vorstand bestellt, ${ }^{6}$ nachdem der

\footnotetext{
${ }^{2}$ Vgl. Internationale Grundsätze für die archivische Verzeichnung. Übersetzt u. bearbeitet v. Rainer BRÜNUNG und Werner HEEGEWALDT. Marburg 1994 (=Veröffentlichungen der Archivschule Marburg. 23); Angelika MENNE-HARITZ (Hg.): Archivische Erschließung: Methodische Aspekte einer Fachkompetenz. Beiträge des 3. Archivwissenschaftlichen Kolloquiums der Archivschule Marburg. Marburg 1999 (= Veröffentlichungen der Archivschule Marburg. 30) u. Nils BRÜBACH (Hg.): Archivierung und Zugang. Transferarbeiten des 34. wissenschaftlichen Kurses der Archivschule Marburg. Marburg 2002 (= Veröffentlichungen der Archivschule Marburg. 36).

${ }^{3}$ Der 514seitige Archivbehelf ist nicht nur im AÖAW einzusehen, sondern auch auf der Homepage des Archivs (http://www.oeaw.ac.at/biblio/Archiv).

${ }^{4}$ Vgl. zu seiner Person den Nachruf von Richard WeTtSTEIN, in: Almanach der Akademie der Wissenschaften in Wien 76 (1926), S. 175-179.

${ }^{5}$ Seit 1943 bestand das ,Vierjahresplaninstitut für Neutronenforschung', das die Kräfte der Wissenschaftler des Instituts für Radiumforschung und des II. Physikalischen Instituts der Universität Wien bündeln sollte. Dem Vierjahresplaninstitut stand der Vorstand des II. Physikalischen Instituts, Georg Stetter, vor, während Gustav Ortner sein Stellvertreter war. Vgl. Berta KARLIK: 1938 bis 1950, in: Sitzungsberichte der math.-nat. Klasse der ÖAW IIa 159 (1950), S. 35-41, hier: S. 36. Im Almanach der Akademie der Wissenschaften in Wien 94 (1944), S. 112, heißt es: „Das Institut [für Radiumforschung] wurde gemeinsam mit dem II. Physikalischen Institut der Universität Wien zum Vierjahresplaninstitut für Neutronenforschung erklärt - mit 1 . April 1944. Zu Direktoren des Vierjahresplaninstituts wurden entsprechend der Zweiheit der Institute Prof. Dr. G. Stetter und Prof. Dr. G. Ortner bestellt. “

${ }^{6}$ Bei Richard MEISTER: Geschichte der Akademie der Wissenschaften in Wien 1847-1947 (Denkschriften der Gesamtakademie. 1) Wien 1947, S. 338, wird Meyer als Vorstand und Karlik als Leiter geführt [analog dazu im Almanach der Akademie der Wissenschaften in Wien 97 (1947), S. 247]. Vgl. zu den ersten Jahren unmittelbar nach Kriegsende: KARLIK, 1938 bis 1950 (wie Anm. 5), S. 39.
} 
bereits 75jährige Meyer mit 1. Oktober 1947 pensioniert worden war. Karlik leitete das Institut als Vorstand und geschäftsführender Direktor bis zum August 1974, ${ }^{7}$ als ihr Herbert Vonach (TU München) nachfolgte. Bis zur Umwandlung im Jahre 1986 leitete dieser das Institut, dessen geschäftsführender Direktor fortan Wolfgang Breunlich war.

Erwähnt seien an dieser Stelle noch die jeweiligen Stellvertreter. ${ }^{8}$ Bis 1920 war Viktor F. Hess sogenannter ,Erster Assistent“ Meyers ${ }^{9}$. Seine Stelle übernahm wegen seiner Berufung nach Graz Karl Przibram, der seinerseits später als „Vorstandstellvertreter" tituliert wurde. Ebenso wie Meyer mußte auch Karl Przibram nach dem Anschluß an Hitler-Deutschland seine Position aufgeben. Als „Funktionäre“ werden in den Almanachen der Akademie in den Kriegsjahren nach dem Leiter Gustav Ortner die „Assistenten“ Friedrich Hernegger und Friedrich Prankl genannt, sowie später auch Erwin Fischer-Colbrie, der aus Nürnberg gekommen war. Berta Karlik wurde als „Dozent mit Diäten“ geführt. Seit April 1945 war Karlik mit der provisorischen Leitung des Instituts betraut, Assistenten waren Hernegger ${ }^{10}$ und wenig später auch Fritz Hawliczek. Friedrich Hernegger war bis 1972/73 wissenschaftlicher Beamter und Oberassistent am Institut; seit 1958/59 wird auch Traude Bernert so bezeichnet (bis 1970/71) und neben diesen beiden seit 1961/62 auch Heinrich Felber. Während unter Karliks Leitung nie explizit von einem Stellvertreter die Rede war, wird seit Beginn der Tätigkeit Herbert Vonachs als Vorstand und geschäftsführender Direktor (1. September 1974) Peter Hille als solcher bezeichnet. Er war Vonachs Stellvertreter bis zur Umwandlung des Instituts für

\footnotetext{
${ }^{7}$ Zum wissenschaftlichen Programm des Instituts vgl. Berta KARLIK: Das Institut für Radiumforschung und Kernphysik, in: Aufgaben der Universität Wien in Gegenwart und Zukunft. Aufsätze zur 600-Jahrfeier. Wien 1965, S. 384-388. Vgl. auch die in den Sitzungsberichten der math.-nat. Klasse der ÖAW II 170 (1961), S. 239-252, abgedruckten Reden, die anläßlich des Festaktes zum 50jährigen Bestehen des Instituts u. a. von Karl Przibram als Obmann des Kuratoriums und Berta Karlik als Leiterin des Instituts gehalten worden waren.

${ }^{8}$ Ein Verzeichnis der wissenschaftlichen Mitarbeiter des Instituts 1910-1950 ist dem Sonderdruck der in Anm. 14 genannten Festschrift beigegeben; fehlt in den Sitzungsberichten.

${ }^{9} \mathrm{Zu}$ seiner Person Gerhard HuEMER: Viktor Franz Hess (phil. Diss. Innsbruck 1985). Zuletzt: Georg FEDERMANN: Viktor Hess und die Entdeckung der kosmischen Strahlung (nat.wiss. Dipl.arb. Wien 2002), bes. S. 82-86 (publiziert unter http:// www.federmann.co.at/vfhess/index.html).

${ }^{10} \mathrm{Vgl}$. Almanach der Akademie der Wissenschaften in Wien 95 (1945), S. 302 f.
} 


\section{Sroblole}

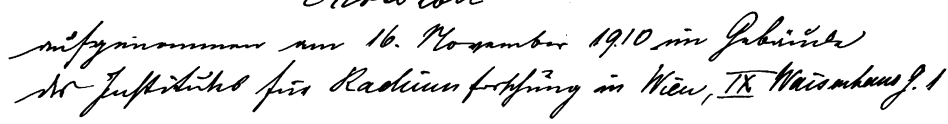

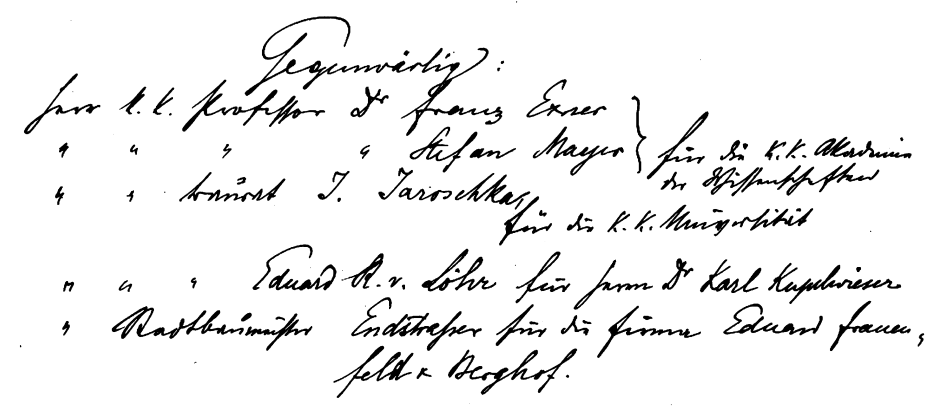

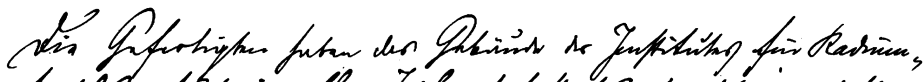

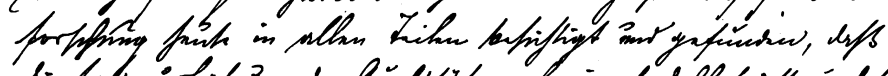

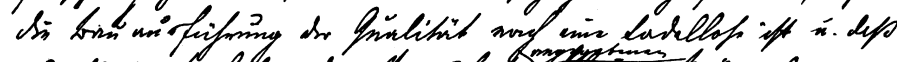

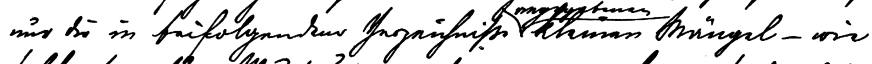

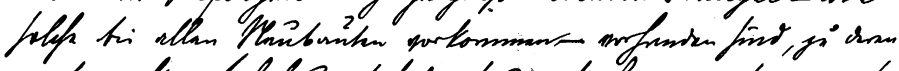

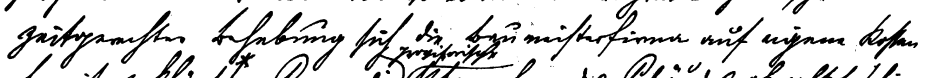

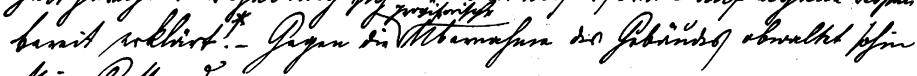
this Cleftrin?
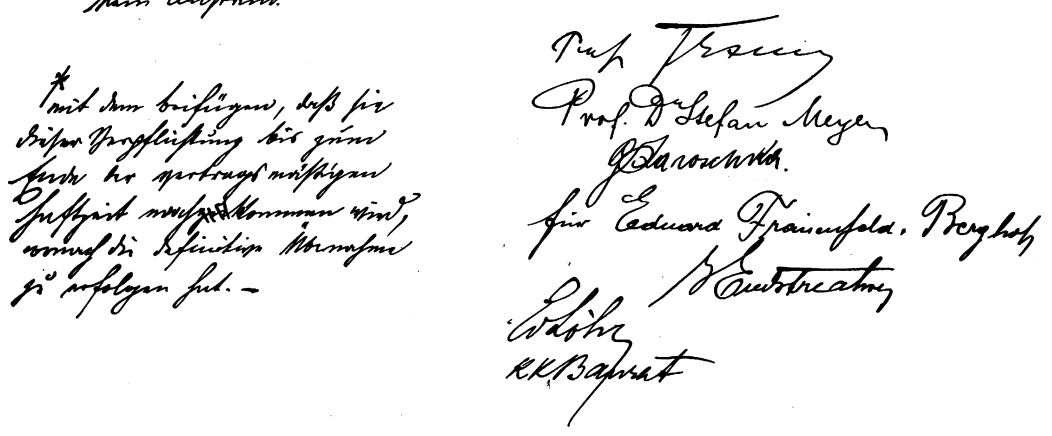

Abb. 1. Übernahmeprotokoll des Gebäudes Boltzmanngasse 3 (Institut für Radiumforschung) am 16. November 1910 (AÖAW, Institut für Radiumforschung, K. 1)

Radiumforschung und Kernphysik in das Institut für Mittelenergiephysik 1986.

Die Forschungsleistungen, die am Institut für Radiumforschung vollbracht wurden, können an dieser Stelle keinesfalls in der 
gebührenden Breite dargelegt werden. Es war das erste Institut dieser Art weltweit und besonders in den Jahren vor 1938 eine anerkannte Größe in der internationalen Atomforschung. Zahlreiche wichtige Entdeckungen wurden hier gemacht und grundlegende Kenntnisse über Radioaktivität gewonnen. Auch in den Kriegszeiten gelangen noch wichtige Entdeckungen, wie die des Elements 85 (Astat) in der Natur durch Berta Karlik und Traude Bernert im Jahre 1944. Von der wissenschaftlichen Tätigkeit des Instituts zeugen die in den Almanachen der Akademie abgedruckten Berichte sowie die Publikationen der Mitarbeiter, von denen zwischen 1911 und 1985 allein 743 als ,Mitteilungen des Instituts für Radiumforschung '(MIR) erschienen. ${ }^{11}$

\section{Geschichte der Akten}

Im Jahre 1956 schrieb Meyers Tochter Agathe Meyer-Rosenqvist aufgrund einer Anfrage an Josip Boncelj, Zagreb, sie habe nur die Korrespondenzen ihres Vaters aus den letzten 20 Jahren bei sich, alles übrige befinde sich beim Institut für Radiumforschung.

Im AÖAW, Archiv-Internes, Institut für Radiumforschung, werden Fotokopien einer älteren Inventarliste mit einer knappen Verzeichnung von Instituts-Akten und „Prof. Karlik privat“ (3 S.) verwahrt, die offenbar bald nach dem Tode Berta Karliks im Jahre 1990 angelegt wurde. Nach handschriftlichen Notizen Lintners wurden später von Akten Karlikscher Provenienz folgende Materialien ausgeschieden bzw. vernichtet: „Deutsche Physikalische Gesellschaft ,Gauverein Österreich“ “, „Lehramtsprüfungskommission“, ,,Studienkommission Physik“, „Selbstbiographie, eigenes Exemplar“", „Kommissionseinladungen" und "Aufzeichnungen von Praktika und Prüfungen“. Unterlagen betreffend den „Verband der Akademikerinnen“ aus der Zeit, als Karlik Präsidentin des Verbandes war, wurden durch Lintner an Frau Mag. Dr. Anna Zemann übergeben. Im selben AÖAWBestand ist auch das Übergabeprotokoll (4 S.) vom 8. Juni 1999 als dem Tag der Übernahme des schriftlichen Materials von der Kommission für die Geschichte der Naturwissenschaften, Mathematik

\footnotetext{
${ }^{11} \mathrm{Vgl}$. auch die Berichte Meyers zum zehn- bzw. fünfundzwanzigjährigen Bestehen des Instituts: Stefan MEYER: Das erste Jahrzehnt des Wiener Instituts für Radiumforschung, in: Jahrbuch der Radioaktivität und Elektronik 17 (1920), S. 1-29, und Stefan MEYER: 25 Jahre Radiuminstitut, in: Anzeiger der Akademie der Wissenschaften in Wien, math.-nat. Kl. 72 (1935), S. 227 f., sowie die unten Anm. 14 zitierte Festschrift zum vierzigjährigen Jubiläum. Zu den MIR vgl. unten Anm. 22.
} 
und Medizin abgelegt. ${ }^{12}$ Der Zustand der Akten war archivtechnisch problematisch. Eventuell vorhanden gewesene Provenienzen waren verwischt worden. Eine gewisse Bearbeitung hatten allein die Korrespondenzen Meyers und Karliks erfahren, wobei die Originale fotokopiert und alles in Klarsichthüllen verpackt in Aktenordnern abgelegt worden war. Die Materialien, die noch gänzlich unbearbeitet waren, ließen die ausgezeichnete Ordnung der ursprünglichen Ablage Meyers erkennen.

\section{Charakterisierung des Bestandes}

Die Ordnung des Materials folgt grundsätzlich folgendem Schema: Auf die Akten der Provenienz ,Zentrale Verwaltung der Akademie“ (I) folgen zunächst allgemeine Akten bezüglich des Instituts (Bau, Status, Mitarbeiter, Finanzen, Tätigkeitsberichte, Kuratorium, $\mathrm{Pu}-$ blikationen, Zeitungsausschnitte, II bis IX) und anschließend nach Nachlässen geordnet Unterlagen aus der Tätigkeit der Institutsvorstände Stefan Meyer (X) und Berta Karlik (XIII). Dazwischen befinden sich in chronologischer Ordnung zunächst einige wenige Unterlagen aus der Zeit der Tätigkeit Gustav Ortners (XI) und der Nachlaß Karl Przibram (XII). Abgeschlossen wird der Bestand mit den Splitternachlässen (XIV), den unvermeidlichen Varia (XV) und schließlich mit den Fotos und Glasplattennegativen. Im Anhang sind die Akten des Vierjahresplaninstituts für Neutronenforschung verzeichnet.

Zunächst werden die Akten der Provenienz ,Zentrale Verwaltung der Akademie" vorgestellt. Diese gehören nicht zu den Akten, die am Institut entstanden sind, und stehen damit außerhalb der übrigen Akten. Dieses Material beschrieb mein Vorvorgänger als Archivar der ÖAW, Dr. Klaus Wundsam, in den 1980er Jahren wie folgt:

Protokolle 1901-1928; Akten Joachimsthal; Bildmaterial von Versuchen; Herstellung von Badezusätzen nach Gasteiner Vorbild; Korrespondenz mit Bädern der Monarchie (Gastein, Teplitz-Schönau, Vöslau); internationale Korrespondenz: RUTHERFORD, P. CURIE, PFAUNDLER, RAMSAY, E. LUDWIG, EXNER, KUPELWIESER, TROWBRIDGE; Abrechnungen;

\footnotetext{
${ }^{12}$ Das Material wurde gemäß Beschluß der Gesamtsitzung der Akademie vom 18. Juni 1999 von der Kommission für Geschichte der Naturwissenschaften, Mathematik und Medizin in das Archiv der Österreichischen Akademie der Wissenschaften (AÖAW) überführt, in dem im Sommer 2000 mit den Verzeichnungsarbeiten begonnen wurde. Dabei waren neben dem verantwortlichen Archivar auch Frau Mag. Dr. Christine Ottner (Nachlaß Przibram), Herr Mag. Dr. Christof Kraus (Nachlaß Karlik), Frau Mag. Yukiko Sakabe (Nachlaß Karlik) und Frau Marianne Wenger an der Erschließung beteiligt. Die Ordnungsarbeiten konnten im Mai 2003 mit der Einschachtelung abgeschlossen werden.
} 
Verzeichnisse; Akten der Radium-Standard-Kommission 1912 ff.; Verrechnungsakten mit England; engl. Briefwechsel: RUTHERFORD, EXNER, MEYER, RAMSAY; Aktenstücke über die Mitteilungen des Instituts für Radiumforschung; Akten über die Verarbeitung von Uranrückständen durch die Österr. Gasglühlicht- und Elektrizitätsgesellschaft in Atzgersdorf (HAITINGER); Statuten des Instituts, Schenkungsurkunde und Gründung des Instituts; Akten über den Neubau des Instituts; Personalien des Instituts; Kaufvertrag mit Lageplan von 1909 (Beckenhausgründe = Boltzmanngasse).

Nach Wundsams Aktenvermerk wurde die Schachtel (damalige Bezeichnung: No. 75) am 7. Sept. 1992 an Frau Dr. Hannelore Sexl übergeben, die jedoch mit Schreiben vom 16. Dez. 2003 angab, dieses Material nie erhalten, sondern vielmehr ihrerseits in den Jahren 1993 und 1996/97 im Archiv vergeblich danach gesucht zu haben. Die hier unter „I. Archiv der ÖAW“ zusammengestellten Akten entstammen zweifelsfrei der zentralen Akademie-Verwaltung; es könnte sich um Relikte der nun als verschollen anzusehenden Akten handeln. ${ }^{13}$ Was bei dem Versuch, dieses Material zu rekonstruieren, sicher diesem urspünglichen Bestand zugeordnet werden konnte, wurde unter „I. Akten des Instituts für Radiumforschung im Archiv der Österreichischen Akademie der Wissenschaften" (1 Mappe) abgelegt. Es handelt sich dabei allein um Akten aus den Jahren 1898 bis 1911, die aufgrund der seinerzeit üblichen und sehr charakteristischen Aktenzahlen in roter Tinte eindeutig als Provenienz ,Zentrale Verwaltung der Akademie" erkannt werden können; weiteres war nicht mehr zu eruieren. Das Material, dessen Schwerpunkt in den Jahren 1904/05 liegt, konzentriert sich auf die Korrespondenz der Akademie mit Marie und Pierre Curie über deren Bemühungen um den Erhalt von Uranpechblenderückständen aus St. Joachimsthal. ${ }^{14}$

\footnotetext{
${ }^{13}$ Erhalten haben sich glücklicherweise jene Akten, die Anfang der 90er Jahre noch nicht in das AÖAW überführt worden waren, sondern in der Verwaltungsstelle der math.-nat. Klasse gelagert wurden. Diese Akten, die - von einigen wenigen Ausnahmen abgesehen - mit Ende des 2. Weltkrieges beginnen, wurden im Jahre 2002 dem AÖAW übergeben. Sie sind mittlerweile ebenfalls in einem eigenen Archivbehelf (27 S.) verzeichnet und als Bestand „,math.-nat. Klasse, Institut für Radiumforschung “ - vorbehaltlich der Bestimmungen der Benutzerordnung des AÖAW, besonders was die Schutzfristen betrifft - einzusehen.

${ }^{14}$ Zwei dieser Briefe hat Stefan Meyer in der ,Festschrift des Instituts für Radiumforschung anläßlich seines 40jährigen Bestandes“ in seinem Beitrag „Die Vorgeschichte der Gründung und das erste Jahrzehnt des Instituts für Radiumforschung “ abgedruckt. Sitzungsberichte der math.-nat. Klasse der ÖAW IIa 159 (1950), S. 1-26, hier: S. 8f. Neben dem Durchschlag des Manuskriptes v. Stefan Meyer (AÖAW, Institut für Radiumforschung, K. 23, Konv. ,Eigene Publikationen') haben sich auch dessen (unpublizierte) Notizen über den Radiumbesitz von Marie Curie zu S. 5 seines Manuskriptes erhalten (ebda), die ,nur privat für Karl [Przibram] und Berta [Karlik]“ bestimmt waren und eine etwas andere Stimmung vermitteln als der gedruckte Text.
} 


\subsection{Allgemeine Akten des Instituts}

Mit „II. Bau des Instituts, Erstausstattung“ beginnt die Reihe der eigentlichen Akten aus dem Institut (1 Mappe). Aus einer größeren Reihe von Kostenvoranschlägen für den Bau und die erste Einrichtung des Instituts aus den Jahren von 1909 bis 1911 und den Bestellungen können die Arbeitsbedingungen am Institut in den ersten Jahren seines Bestehens rekonstruiert werden, wenngleich man bei der Auswertung dieser Unterlagen stets eine mögliche Unvollständigkeit einkalkulieren muß. Eine Überprüfung der Vollständigkeit könnte allerdings mittels der Kostenzusammenstellungen Meyers aus den Jahren 1909/10 gelingen. Im Zusammenhang mit dieser Fragestellung sind auch die Akten „V. Finanzen“ von kaum zu überschätzender Bedeutung (s. u.).

Von stetem Interesse ist der „III. Status des Instituts“ (1 Mappe) gewesen. Berta Karlik hat im Jahre 1977 versucht, die Stellung des Instituts als Akademiegründung, das regelmäßige Dotationen durch das Ministerium erhielt und deren Mitarbeiter zumindest teilweise (auch) Lehraufträge an der Universität Wien wahrnahmen, darzulegen. Für ihre (interne) Darstellung hat sie teilweise originale Akten aus dem Institut verwendet, die gemeinsam mit ihren eigenen Notizen in dieser Zusammensetzung beibehalten wurden.

Unter „IV. Mitarbeiter“ (1 Mappe) wurden personenbezogene Unterlagen abgelegt, d. h. sowohl zu den wissenschaftlichen Mitarbeitern des Instituts als auch zu den Mechanikern und Institutsdienern. ${ }^{15}$ Der Schwerpunkt des Materials liegt in der Vorkriegszeit. Während die Jahre von 1938 bis 1945 aktenmäßig überhaupt nur schlecht dokumentiert sind, fallen unter den Personalia zwei Fälle besonders auf: Zum einen befindet sich unter den Akten eine Stellungnahme der NSDAP vom 8. April 1941 zur politischen Gesinnung Berta Karliks, in der es u. a. heißt: ,,politisch ist sie uninteressiert und daher in keiner Weise hervorgetreten. Irgendwelche Bedenken in politischer Beziehung sind nicht zu erheben“"16; zum anderen eine Bescheinigung vom 1. August 1945 für Traude Bernert, in der ihre ,politische Unbedenklichkeit' bestätigt wird. ${ }^{17}$

\footnotetext{
${ }^{15}$ Siehe oben Anm. 8.

${ }^{16}$ AÖAW, Institut für Radiumforschung, K. 1, Konv. ,Mitarbeiter', Mappe ,Karlik, Berta'.

17 „Dr. Traude Bernert ist durch dieses Amt [Geheimpolizei-Korps, Abt. Oberösterreich, Unterabt. Vöcklabruck] verhört und geprüft worden und es wurde in Bezug auf ihre politische Anschauung und Tätigkeit nichts Nachteiliges ermittelt". AÖAW, Institut für Radiumforschung, K. 1, Konv. ,Mitarbeiter', Mappe ,Bernert, Traude‘.
} 


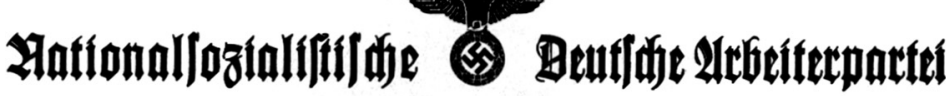

\section{Bauleitung 201ien}

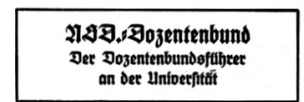

Dozentenbundoffilyte:

an Det Uniperfitát
An das

Dekanat der Philosoph. Fakultät

W $i$ e $n$ I/ 1

Universität

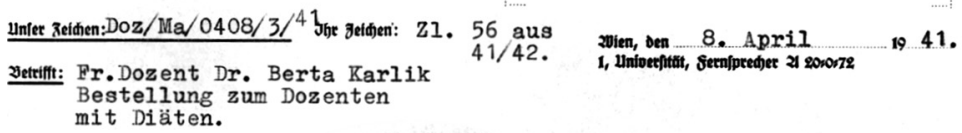
mit Diäten.

wien, den 8. April

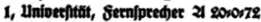

19.41.

Frau Dozent Dr. Berta $K$ a $r l i k$ ist charakterlich absolut einwandfrei. Sie ist ein repräsentativer Mensch, der persönlich sehr wohl geeignet ist als Hochschullehrer zu wirken. Politisch ist sie uninteressiert und daher in keiner Weise hervorgetreten. Irgendwelche Bedenken in politischer Beziehung sind nicht $z u$ erheben. Die Gewährung von Diäten für die Dozentin Dr. Berta $\mathrm{K}$ a $\mathrm{r} I \mathrm{i} k$ wird daher befurwortet.

Heil H i t le r !

Der Dozentenführer der Universität:

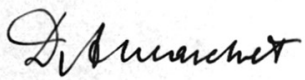

(Dr. A. Marchet)

Abb. 2. Stellungnahme der NSDAP zur Bestellung Berta Karliks zum Dozenten mit Diäten vom 8. April 1941 (AÖAW, Institut für Radiumforschung, K. 1)

Es bedarf noch einer näheren Beschäftigung mit den „V. Finanzen“ (3 $1 / 2$ Kartons) ${ }^{18}$ des Instituts, um das System der Finanzierung und der hausinternen Buchhaltung zu durchschauen. Das Institut wurde einerseits mit Dotationen durch das Ministerium und andererseits durch die Akademie finanziert. Gegenüber beiden Einrichtungen wurden Rechnungslisten präsentiert, wofür die jeweils zur Verfügung gestellten Mittel verwendet wurden. Während aus den Rechnungslisten gegenüber dem Ministerium nur die Namen des Lieferanten und die

\footnotetext{
${ }^{18}$ AÖAW, Institut für Radiumforschung, K. 2-5.
} 
Rechnungssumme hervorgehen (vorliegend für die Jahre von 1912 bis 1940), sind jene gegenüber der Akademie (vorliegend für die Jahre von 1910 bis 1942 sowie einige wenige Unterlagen bis 1973) deutlich aussagekräftiger, da sich überwiegend auch die Belege erhalten haben. In diesen Fällen kann exakt die gelieferte Ware festgestellt und durch den Erwerb dauerhaft verwendbarer Gerätschaften und Verbrauchsmaterialien indirekt der Schwerpunkt der alltäglichen Arbeit am Institut erschlossen werden.

Aber auch die scheinbar bedeutungslosen Unterlagen der sogenannten „Institutsverrechnung “ erlauben interessante Einblicke, zumal auch hier nicht nur die Rechnungsbücher für die Jahre von 1922 bis 1938 vorliegen, sondern des weiteren zahlreiche Einzelbelege aus den Jahren von 1934 bis 1938. Sie sind notwendig, um Eintragungen in den Rechnungsbüchern verstehen zu können. So wird deutlich, daß die unregelmäßigen Zahlungen an den Institutsmechaniker Karl Kornher keine zusätzliche Besoldung darstellen, sondern schlicht Rückerstattungen von Auslagen. Kornher war also nicht allein für mechanische Arbeiten am Institut verantwortlich, sondern hat offenbar auch immer wieder kleinere Erledigungen und Besorgungen für das Institut gemacht, wobei er die Beträge stets vorstreckte.

Es finden sich jedoch rund 220 weitere Rechnungen aus den Jahren von 1910 bis 1973, die offenbar weder dem Ministerium noch der Akademie zur Verrechnung vorgelegt wurden, zumal sich auch nicht alle Rechnungen in den Rechnungsbüchern der „Institutsverrechnung “ wiederfinden. Hier müssen erst detaillierte Untersuchungen die Finanzgebarung des Instituts aufarbeiten. Für die Kriegsjahre haben sich verbrauchte Scheckhefte und Überweisungshefte von August bzw. Juni 1940 bis März 1945 erhalten, in denen sich Zusammenstellungen über die Verwendung der Schecks und der Überweisungen befinden.

Die „VI. Tätigkeitsberichte“ (1 Mappe) des Instituts haben sich für die Jahre von 1939 bis 1962 erhalten. Sie schließen deutlich mehr ein als nur die Manuskripte für die jährlichen Berichte in den Almanachen der Akademie. Aufschlußreich für die Arbeiten am Institut in den Jahren von 1953 bis 1956 sind vor allem die detaillierten monatlichen Berichte an den Elektrotechnischen Verein Österreichs (Dr. Friedrich C. Saic), dem regelmäßig über den Aufbau eines Szintillationsspektrometers (seit Herbst 1953) berichtet wurde, wodurch dessen Fertigstellung exakt dokumentiert ist. Auch über die wissenschaftlichen Arbeiten am Neutronengenerator und - seit Anfang 1956 - am Szintillationsspektrometer wurden dem Elektrotechnischen Verein bis Dezember 1956 ausführliche Berichte übermittelt. ${ }^{19}$

\footnotetext{
${ }^{19}$ AÖAW, Institut für Radiumforschung, K. 5.
} 
Das „VII. Kuratorium des Instituts für Radiumforschung“ (1 $1 / 2$ Kartons) ging aus der 1901 eingesetzten „Kommission für die Erforschung radioaktiver Substanzen" hervor und wurde am 28. April 1938 konstituiert. Am Institut selbst lagen offenbar keinerlei Akten zu den Kuratoriumssitzungen vor; die hier zusammengestellten Akten entstammen ganz überwiegend dem Nachlaß Karlik. „Die Akademie hat für jedes Institut ein Kuratorium zu bestellen. [...] Das Kuratorium hat die Aufgabe, die Akademie bei der Aufsicht über das Institut zu unterstützen"; so legt es die heute gültige Fassung der Geschäftsordnung der Akademie fest. ${ }^{20}$ Dementsprechend gehören zu den Unterlagen einer Sitzung in aller Regel das vorläufige Protokoll der letzten Sitzung, der Bericht über das vergangene Jahr und die Arbeitsvorhaben für das kommende Jahr sowie die das Budget betreffenden Akten. Eine kontinuierliche Reihe beginnt erst im Jahre 1972 und reicht bis zu den ersten beiden Sitzungen des aus dem Institut für Radiumforschung hervorgegangenen Instituts für Mittelenergiephysik $1988 .^{21}$ Für die früheren Jahre haben sich ab 1947 nur einige wenige Unterlagen erhalten.

Unter den „VIII. Publikationen des Instituts (MIR)“ (1 1/2 Kartons) befindet sich naturgemäß wenig unbekanntes Material, da die Erträge der Forschungsleistung in diese „Mitteilungen des Instituts für Radiumforschung " (MIR) mündeten, die - anders als die übrigen in diesem Archivbestand vorhandenen Unterlagen - eben keine Unikate sind. Die MIR sind bibliographisch erschlossen ${ }^{22}$ und in jeder

\footnotetext{
${ }^{20}$ Geschäftsordnung der Österreichischen Akademie der Wissenschaften, zuletzt geändert gemäß Beschluß der Gesamtsitzung am 11. Mai 1993, Abs. VI B, § 53(1) bzw. 54(1). Erst seit dem Jahre 1976 müssen gemäß Geschäftsordnung Instituten verpflichtend Kuratorien beigegeben werden.

${ }^{21}$ Das Protokoll der außerordentlichen Kuratoriumssitzung vom 4. März 1986 befindet sich neben etlichen in den Zusammenhang der Umwandlung des Instituts gehörenden Papieren unter den thematischen Korrespondenzen im Nachlaß Karlik. AÖAW, Institut für Radiumforschung, K. 57.

${ }^{22}$ Zwischen 1911 und 1985 erschienen insgesamt 743 MIR. Ein Verzeichnis 1911-1970 (No. 1-633a) ist abgedruckt in „Dokumentation zur Österreichischen Akademie der Wissenschaften 1847-1972“, Bd 2: Die Schriften der mathematischnaturwissenschaftlichen Klasse, Wien 1972, S. 474-503; ein Verzeichnis 1971-1985 (No. 634-743) ist in derselben Dokumentation, Bd 4: Die Schriften der mathematisch-naturwissenschaftlichen Klasse 1971-1996, Wien 1998, S. 295-304, abgedruckt. Eine vollständige Reihe der MIR bis 1973 (No. 664b) in 24 Bänden befindet sich unter der Sign. 15.980-B.AR im Bestand der Bibliothek der ÖAW. Von den zwischen 1908 und 1911 als Vorläufer der MIR erschienenen acht Mitteilungen der Radium-Kommission befindet sich ein gebundenes Exemplar (Fotokopien) unter der Sign. 30886-C.AR im Bestand der Bibliothek der ÖAW; diese acht Hefte sind in Band 2 der o. g. Dokumentation, S. 474, bibliographisch erfaßt.
} 
größeren Bibliothek einzusehen. Von den 743 Einzelpublikationen liegen zu 219 aus den Jahren 1911 bis 1979 Manuskripte, Druckfahnen oder Sonderdrucke vor. Wenn derartige Unterlagen zu den MIR in den Nachlässen von Stefan Meyer, Karl Przibram oder Berta Karlik gefunden wurden, so sind sie hier unter dem genannten Punkt VIII abgelegt. Aufschluß über die bekanntermaßen weitreichenden wissenschaftlichen Kontakte des Instituts gibt ein Verzeichnis von „Adressen für die Zusendung von Separaten von Instituts wegen“, 23 in dem bis 1927/28 genau verzeichnet ist, welchen Personen jeweils eine Nummer der MIR übersendet wurde. Der erste Name in diesem Verzeichnis ist jener von Karl Kupelwieser.

Unter den Akten befand sich auch eine größere Anzahl von „IX. Zeitungsausschnitten“ (1/2 Karton Originale und 1/2 Karton Fotokopien), ${ }^{24}$ für die jedoch im Einzelfall nicht mehr festzustellen war, auf welchem Wege sie an das Institut gelangten; teilweise scheinen sie systematisch gesammelt worden $\mathrm{zu}$ sein, teilweise wurden sie von ausländischen Forscherkollegen übersandt. Immerhin läßt sich für die überwiegende Zahl die Zeitung nennen, aus der der jeweilige Ausschnitt stammt. Die Zeitungsausschnitte wurden chronologisch geordnet, wobei 228 aus den Jahren von 1909 bis 1986 mit Autor (sofern angegeben), Titel, Publikationsorgan und Datum ausführlich verzeichnet werden konnten. Weitere 22Zeitungsausschnitte waren nicht $\mathrm{zu}$ datieren. Inhaltlich wurde offensichtlich alles gesammelt, was in einem Zusammenhang mit der Radium- und später der allgemeinen Atomforschung stand: Berichte über Tagungen (,I. Radiumkongreß in Brüssel“", in: Pharmazeutische Post v. 2. Nov. 1909), über die Gründung weiterer Radium-Forschungsstätten (,Errichtung eines staatlichen radiologischen Institutes in St. Petersburg “, in: Deutsche Tageszeitung, Berlin, v. 6. Mai 1914 und in: Kölnische Zeitung v. 8. Mai 1914), über die positiven und negativen gesundheitlichen Folgen von Radioaktivität (,Das Radium als Heilfaktor“", in: Die Zeit v. 24. Okt. 1909 und „Radiumforscher Professor Fernau. Opfer der Wissenschaft. Geheimnisvolle Leuchterscheinung an dem Toten. Schicksalstragödie eines Wiener Gelehrten“", in: Neues Wiener Journal v. 13. Sept. 1934) bis hin zu personenbezogenen Meldungen (,Prof. Boltwood of Yale killed himself“, in: The Boston Daily v. 16. Aug. 1927 und „Eine verdiente Ehrung [Ernennung Berta Karliks zum korrespondierenden Mitglied

\footnotetext{
${ }^{23}$ AÖAW, Institut für Radiumforschung, K. 8.

${ }^{24}$ Wegen der starken Übersäuerung besonders der älteren Zeitungsausschnitte werden dem Benutzer nur die Fotokopien vorgelegt.
} 
der Österreichischen Akademie der Wissenschaften]", in: Die Frau v. 24. Juli 1954) oder der Diskussion um den Bau eines Atomreaktors in Österreich (,Ein Atomreaktor für Österreich in Erwägung “, in: Die Presse, Wien, v. 12. Jan. 1955) und die Errichtung des Forschungsreaktors in Seibersdorf (,Atome für den Frieden“, Sonderbeilage des Neuen Österreich v. 27. Sept. 1960 anläßlich der Inbetriebnahme des Reaktors).

\subsection{Nachlaß Stefan Meyer}

Von zentraler Bedeutung innerhalb des Bestandes „Institut für Radiumforschung“ steht der „X. Nachlaß Stefan Meyer“ (23 1/2 Kartons). Hierbei handelt es sich um einen rein wissenschaftlichen Nachlaß, d. h. um jene Papiere, die aus seiner Tätigkeit als Leiter oder Vorstand des Instituts für Radiumforschung hervorgegangen sind. ${ }^{25}$ Es finden sich keinerlei persönliche Dokumente über Meyers Schulzeit (Zeugnisse o. ä.), Aufzeichnungen aus seiner Studentenzeit (Vorlesungsmitschriften, Prüfungszeugnisse oder Diplome) oder Korrespondenzen oder Fotos seiner Familie. Wohl eher zufällig hat sich eine Empfangsbestätigung der Gesellschaft der Musikfreunde in Wien vom 22. September 1869 hierher verirrt, die 200 fl. ,,als einzige Rate des Gründungsbeitrages “ durch Meyers Vater Gotthelf Karl Meyer (1844-1905) ausweist. ${ }^{26}$ Einige wenige weitere Unterlagen aus den letzten Jahren vor seinem Tod sind wohl aufgrund der Bemühungen Karliks um die Person Meyers zu diesen Akten gelangt.

Die Arbeit Stefan Meyers am Institut, das er - wie einleitend erwähnt - seit der Gründung 1910 leitete, ist hingegen ausgezeichnet, vor allem durch die Korrespondenzen, dokumentiert. ${ }^{27}$ Bei der

${ }^{25} \mathrm{Zu}$ Meyers Biographie: Karl PrZIBRAM: [Nachruf auf] Stefan Meyer, in: Almanach der Österreichischen Akademie der Wissenschaften 100 (1950), S. 340-352. Wolfgang L. REITER: Österreichische Wissenschaftsemigration am Beispiel des Instituts für Radiumforschung der Österreichischen Akademie der Wissenschaften, in: Friedrich Stadler (Hg.): Vertriebene Vernunft II. Emigration und Exil österreichischer Wissenschaft. Internationales Symposion 19. bis 23. Oktober 1987 in Wien. Wien-München 1988, S. 709-729, hier: S. .709-715. Wolfgang L. REITER: Stefan Meyer und die Radioaktivitätsforschung in Österreich, in: Anzeiger der phil.-hist. Klasse [der Österreichischen Akademie der Wissenschaften] 135 (2000), S. $105-143$.

${ }^{26}$ AÖAW, Institut für Radiumforschung, K. 9.

${ }^{27}$ Mit der Erstellung des Archivbehelfes ist Berta KARLIK: Der wissenschaftliche Briefwechsel von Stefan Meyer, in: Sitzungsberichte der math.-nat. Klasse der ÖAW II 188 (1979), S. 219-230, überholt. 
Verzeichnung der Korrespondenzen wurde versucht, zwischen Meyers Schriftverkehr als Institutsvorstand (,,[Privat-]Korrespondenzen als Vorstand des Instituts“", Ia) und jenem, den er in seiner Eigenschaft als Sekretär (und späterer Präsident) der Radium-Standard-Kommission (,,Korrespondenzen als Sekretär bzw. Präsident der Radium-StandardKommission“, Ib) geführt hat, zu unterscheiden. Diese Trennung birgt freilich Probleme in sich, da die Briefwechsel mit einzelnen Wissenschaftlern kaum streng der einen oder anderen Gruppe zugeordnet werden konnten. Gleichwohl wurde der verantwortungsvollen Aufgabe Meyers in der Radium-Standard-Kommission dadurch Rechnung getragen; ${ }^{28}$ sein Schriftverkehr mit einzelnen Fachkollegen wurde geschlossen der einen oder anderen Kategorie zugeordnet und im Repertorium durch Querverweise entsprechend gekennzeichnet. ${ }^{29}$ Zur Ordnung der Korrespondenzen ist festzuhalten, daß sie in alphabetischer Reihenfolge nach dem Familiennamen der Partner angelegt sind. Sofern sich ein Name im Verlauf des Schriftverkehrs geändert hat, wurde im Archivbehelf ein entsprechender Querverweis gesetzt. Wenn ein Korrespondenzpartner Meyers in seiner Eigenschaft als Leiter einer befreundeten Forschungseinrichtung geschrieben hat, wurden diese Briefe unter dem Ort dieser Forschungseinrichtung abgelegt. So findet sich beispielsweise

\footnotetext{
${ }^{28}$ Zur Tätigkeit der Radium-Standard-Kommission und dem Problem der primären und sekundären Radium-Standards vgl. den von Stefan MEYER gemeinsam mit Otto HAHN und Erwin VON SCHWEIDLER publizierten Bericht über die Versammlung der internationalen Radium-Standard-Kommission in Paris vom 25. bis 28. März 1912, in: Physikalische Zeitschrift 13 (1912), S. 524 f. Außerdem: Stefan MEYER: Die radioaktiven Konstanten nach dem Stand von 1930. Bericht der Internationalen Radium-Standard-Kommission, in: Physikalische Zeitschrift 32 (1931), S. 569-581 (hierzu vorbereitende Unterlagen im AÖAW, Institut für Radiumforschung, K. 22); Stefan MEYER: Über die Radium-Standard-Präparate, in: Anzeiger der Akademie der Wissenschaften in Wien, math.-nat. Kl. 82 (1945), S. 25-30, u. Otto HöNIGSCHMID $(\dagger)$ : Geschichte und Herstellung des primären Radium-Standards, in: ebda, S. 30-34. Zur Kontroverse mit Carlfriedrich Weiss vgl. die im AÖAW, Institut für Radiumforschung, K. 31, vorhandenen 45 Briefe aus den Jahren 1941-1943. Das Konvolut enthält überwiegend Korrespondenzen der Provenienz Gustav Ortner; dessen Briefwechsel m. Weiss u. Meyer, sowie Meyers m. Hönigschmid u. Hahn, außerdem das „Resumé des Entrevues“ von I. Joliot-Curie, Joliot, Weiss, van Stappen u. Kipfer im Collège de France, Paris (2.-5. Juni 1943). Vgl. auch die Briefe Meyers an Karlik v. 8. Juli 1942 (AÖAW, Institut für Radiumforschung, K. 45, Originalbrief im Nachlaß Karlik), 3. Okt. 1942 (ebda, K. 14, Durchschlag im Nachlaß Meyer) u. 24. Mai 1943 (ebda, K. 14, Durchschlag im Nachlaß Meyer, u. K. 45, Originalbrief im Nachlaß Karlik).

${ }^{29} \mathrm{Im}$ Archivbehelf sind Querverweise zur leichteren Erkennung grundsätzlich kursiv gesetzt.
} 
der Briefwechsel mit Ferdinand Scheminzky aus den Jahren von 1935 bis 1938 unter „Badgastein, Forschungsinstitut" und jener ab 1945 unter dessen Namen (mit entsprechendem Querverweis). Grundsätzlich gilt, daß Kontakte generell eher als dienstlich angenommen werden und bevorzugt unter dem Namen der Dienststelle (,Dienstort, Dienststelle“) zu finden sind. In anderen Fällen steht die Person im Vordergrund, wie bei Samuel Colville Lind, der für verschiedene staatliche Stellen in den USA tätig war, und dessen Schreiben unter seinem Familiennamen zu finden sind (auch hier natürlich mit entsprechenden Querverweisen unter „Washington, Department of the Interior. Bureau of Mines", „Denver/CO, Department of the Interior. Bureau of mines experiment Station“ oder „Reno/Nevada, Department of the Interior. Bureau of mines experiment Station"). Die Ordnung nach Orten hat auch für Zeitschriften und Verlage Gültigkeit. So findet sich Meyers Briefwechsel mit der Verlagsbuchhandlung Vieweg \& Sohn unter „Braunschweig, Verlagsbuchhandlung Vieweg \& Sohn“ und jener mit der Verlagsbuchhandlung Johann Ambrosius Barth unter „Leipzig, Verlagsbuchhandlung Barth“.

Die Jahre von 1938 bis 1945 sind - wie schon mehrfach angedeutet auch im Nachlaß Stefan Meyer nur schwach dokumentiert, was in diesem Fall immerhin mit Meyers Abwesenheit vom Institut hinlänglich erklärt werden kann. Da es nicht sehr zielführend gewesen wäre, einzelne fortgeführte Agenden mit dem Ausscheiden Meyers abbrechen und mit Ortner neu beginnen zu lassen, wurden einige Schriftwechsel bis in die 40er Jahre hinein unter dem Nachlaß Stefan Meyer abgelegt, obwohl dieser nicht mehr dafür verantwortlich war. $\mathrm{Zu}$ denken ist hier beispielweise an den Apparate-Ausschuß der Notgemeinschaft der Deutschen Wissenschaft [unter „Berlin, Notgemeinschaft der Deutschen Wissenschaft (Apparate-Ausschuß)“], dessen Schriftverkehr bis 1941 vorliegt, oder an die Treibacher Chemischen Werke (unter ,Treibach, Treibacher Chemische Werke“, bis 1948).

Insgesamt liegen Briefwechsel Meyers mit $696(665+31)$ Korrespondenzpartnern vor. Mehr als 100 Schriftstïcke, d. h. Originalbriefe der Absender und Konzepte oder Durchschläge Meyers, liegen von Hans Benndorf (354), Otto Hönigschmid (233), Viktor Hess (202), ${ }^{30}$

\footnotetext{
${ }^{30}$ Einen beträchtlichen Anteil dieser Korrespondenz hat HUEMER, Hess (wie Anm. 9), S. 158-349, wiedergegeben; vgl. hierzu dessen diesbezügliche Vorbemerkungen (ebda, S. 136-157).
} 
Marie Curie (147), Ernest Rutherford (139), ${ }^{31}$ Egon von Schweidler (138), Hans Pettersson (134), Fritz Paneth (123), Georg Hevesy (116) und Otto Hahn (111) vor. Auch unter diesen sind die Jahre von 1938 bis 1945 in aller Regel schwach bis gar nicht vertreten. Eine erfreuliche Ausnahme stellen die Briefwechsel mit Benndorf, Hönigschmid und Hahn dar. Mit den vor Ort ansässigen Mitarbeitern wurde in aller Regel natürlich nicht schriftlich kommuniziert - bzw. nur dann, wenn sie sich außerhalb Wiens aufhielten -, so daß die vorhandenen Briefe bevorzugt die nationalen und internationalen Kontakte Meyers und des Instituts aufzeigen. Über den Tod Meyers hinaus hat dessen Tochter Agathe (Koss-)Rosenqvist einzelne Korrespondenzen fortgeführt, so zum Beispiel mit Robert W. Lawson (bis 1950), Georg Hevesy (bis 1952), mit Ferdinand Scheminzky (bis 1955) und mit Samuel Colville Lind (bis 1957). Diese wurden im Anschluß an die jeweiligen Korrespondenzen Meyers gereiht. Mit Hans Benndorf führte Agathe (Koss-)Rosenqvist zwischen 1941 und 1949 einen eigenständigen Briefwechsel, der immerhin 16 Schreiben umfaßt.

Zeugt schon allein die Quantität der Korrespondenz Meyers mit Benndorf von deren engem Verhältnis zueinander, so klärt ein Blick in den Briefwechsel schnell, daß es zwischen ihnen nicht allein um das rein Fachliche, sondern auch immer wieder um den Austausch persönlicher Meinungen, Einschätzungen und Beobachtungen ging. Das Augenzwinkern ist unübersehbar, wenn Benndorf am 27. Februar 1932 förmlich ,an die Direktion des Institutes für Radiumforschung ““ schreibt, während er Meyer sonst mit „Lieber Freund“, ,Lieber St.“ oder ,Lieber M.“ anredet:

Der unterzeichnete Leiter des Physikalischen Institutes erlaubt sich diensthöflichst folgende Frage an das Institut für Radiumforschung zu richten. Im Physikalischen Institut der Universität Graz werden schon seit über einem Jahr Messungen des Emanationsgehaltes von Kellerluft ausgeführt, wobei sich ein Emanationsgehalt von etwa $10^{-14}$ Curie $/ \mathrm{cm}^{3}$ ergab. Mit Rücksicht auf die in einem Salzburger Bergwerk gemachten Erfahrungen, wird angefragt, ob nach wohldortiger Erfahrung auch für Versuchspersonen, die sich nicht unmittelbar im emanationshältigen Raume, sondern in einem Stockwerke darüber befinden, Masturbationsgefahr besteht, und durch welche Mittel eine solche ungünstige Beeinflussung der studierenden Jugend hintangehalten werden könnte. Es wird hinzugefügt, daß es sich um Personen

\footnotetext{
${ }^{31}$ Die Briefe, die Otto HitTMAIR: Ernest Rutherford und das Wiener Radiuminstitut. Ein Kommentar zu einem Briefwechsel, wesentlich Marie Curie betreffend, mit dem Institutsdirektor Stefan Meyer, in: Sitzungsberichte der math.-nat. Klasse der ÖAW II 211 (2002), S. 175-190, für seine Darstellung der Beziehungen zwischen Meyer und Rutherford in den Jahren 1911/12 verwendet hat, befinden sich - entgegen den dortigen Angaben (S. 175 u. 179) - nicht im AÖAW.
} 
(62)

\section{FAGULTÉ DES SGIENGES DE PARIS}

\section{12, Rue Cuvier}

LABORATOIRE

de

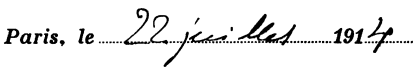

PHYSIQUE GÉNÉRALE

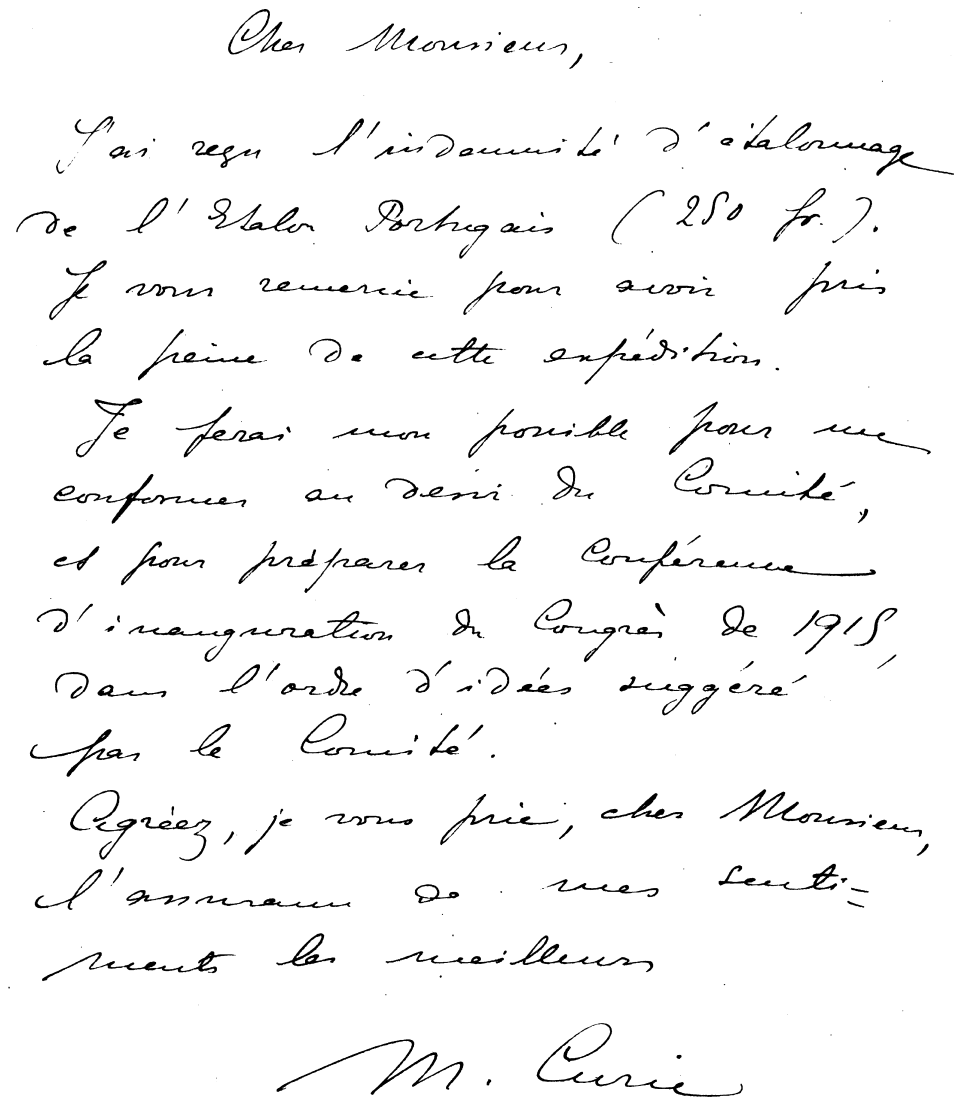

Abb. 3. Eigenhändiger Brief Marie Curies an Stefan Meyer vom 22. Juli 1914 (AÖAW, Institut für Radiumforschung, K. 22) 
beiderlei Geschlechtes, aber in getrennten Räumen, im Alter von 18 - 22 Jahren handelt. ${ }^{32}$

\section{Meyer antwortete am 1. März mit angemessenem Ernst:}

In diensthöflicher Beantwortung Ihrer geschätzten Anfrage vom 27. II. erlaube ich mir mitzuteilen, daß die ,Dobermann-Reaktion“ bisher noch nicht hinreichend studiert wurde, um verlässlich angeben zu können, für welche Em-Gehalte das Maximum auftritt und ob dieses Maximum steilen oder flachen Verlauf hat. Dementsprechend wird es Ihrer Erwägung anheimgegeben, ob nicht mit der durchgreifenden Sterilisierung aller in Frage kommenden männlichen bzw. dobermännlichen und doberweiblichen Personen zuzuwarten wäre, bis anlässlich der Fachtagung am 7. März a.c. eine eingehende Aussprache erfolgen kann. Hochachtungsvollst Stefan Meyer, Vorstand und Krampfmeister des Institutes für radioaktive Sorgen. ${ }^{33}$

Der entspannte Umgangston findet sich auch in weiteren Korrespondenzen Meyers. In Vorbereitung der Feierlichkeiten zum 60. Geburtstag Benndorfs am 13. Dezember 1930 berichtet dessen Assistent Fritz Kohlrausch am 18. Oktober an Stefan Meyer: „13. XII. 1930, Benndorf: Bdf [=Benndorf] hätte eigentlich nach Wien fahren sollen, was wir hinterrücks verhindert haben. [...] Nachmittag findet im Benndorfinstitut ein Festtee statt. [...] Von einem Festvortrag in ernster oder heiterer Form haben wir aus Gründen der Zwanglosigkeit abgesehen. Dafür soll Abends in irgend einem Lokal ein gemeinsames Abendessen stattfinden, [...]. Bei diesem Abendessen bzw. nachher soll an Bdf die gebundene Bibel und der Wanderbecher unter geeigneten Ansprachen überreicht werden, die Ihr alten Kracher übernehmen sollt. Ferner sollen die eingelaufenen ,humorvollen Beiträge ' in geeigneter Form verzapft und der Film in Form eines Traumes, den Bdf auf der Fahrt nach Wien zur Akademiesitzung hatte, vorgeführt werden. [...] Wanderbecher wird von uns Grazern besorgt. " ${ }^{34}$ Bei dem angesprochenen Wanderbecher handelt es sich um den sogenannten Exner-Becher, der seit 1930 unter den Schülern Franz Exners kursierte und an den nächsten 60jährigen weitergegeben wurde. Zuletzt erhielt ihn 1947 Erwin Schrödinger; der Becher wird heute im Archiv der ÖAW aufbewahrt. ${ }^{35}$

\footnotetext{
${ }^{32} \mathrm{AÖAW}$ Institut für Radiumforschung, K. 10.

${ }^{33}$ Ebda, umseitig.

${ }^{34}$ AÖAW, Institut für Radiumforschung, K. 15.

${ }^{35}$ Vgl. Berta KARLIK u. Erich SCHMID: Franz S. Exner und sein Kreis. Ein Beitrag zur Geschichte der Physik in Österreich. Wien 1982, S. 106 (m. Abb.). Zur Korrespondenz Meyer - Schrödinger ist auf das (unvollständige) Stückverzeichnis bei Berta KARLIK: Register der Briefe von Erwin Schrödinger an Stefan Meyer, in: Anzeiger der math.-nat. Kl. der ÖAW 124 (1987), S. 71, hinzuweisen.
} 
Mehr als vierzig Schriftstücke liegen vor an Korrespondenzen mit Carl Ulrich (90), Robert W. Lawson (82), Fritz Kohlrausch (77), Irène Curie-Joliot (66), Samuel Colville Lind (65), Heike KamerlinghOnnes (52), Friedrich von Lerch (52), Lise Meitner (51), ${ }^{36}$ Carl Auer von Welsbach (49), Berta Karlik (44), Elisabeth Rona (43) und Marietta Blau (43).

Neben dem nach Korrespondenzpartnern geordneten Schriftverkehr bestehen auch einige Konvolute thematischer Akten, in denen sich gleichfalls Briefe befinden. Diese thematischen Akten wurden offenbar noch durch Meyer selbst zusammengestellt, weswegen davon abgesehen wurde, die überlieferte Ordnung aufzuheben. So sind beispielsweise Mappen vorhanden ,Zur Kenntnis des Aufenthaltes radioaktiver Producte aus dem Besitz der k. Akademie d. Wiss." (1898 bis in die 1940er Jahre), über den Streit um die Lehrkanzlei Jäger an der Universität Wien (1933-1936) oder wegen der Radiumbeschaffung für Hans Pettersson (1931), aber auch zu Anfragen wegen Institutsbesichtigungen (1916-1926). ${ }^{37}$ Analoges gilt für die „Älteste Aktenverwaltung Meyers“338, die er offenbar sehr bald nach Gründung des Instituts anlegte und bis in die späten 20er bzw. die frühen 30er Jahre führte. In 22 kleinen Einheiten finden sich Stichworte wie ,[Instituts-]Gebäude“, ,Entlehnungen“, ,,Alkohol, Aether“, aber auch „Zollplackereien“, „Unnützes“ oder „Überflüssige oder nicht aktuelle Akten".

Bei den vorhandenen Publikationen wurde unterschieden, ob es sich um solche handelt, bei denen Meyer als alleiniger Autor in Erscheinung trat (,eigene Publikationen“) oder als Ko-Autor (,gemeinsame Publikationen“), bzw. um solche, die ihm von dritter Seite zur Verfügung gestellt wurden (,fremde Publikationen“). Die ersten beiden Kategorien sind chronologisch geordnet, die dritte nach Autoren. Unter diesen Publikationen Meyers sind in erster Linie Separata zu finden; gelegentlich vorhandene Manuskripte oder Druckfahnen sind den Separata beigelegt. Von den im Werkverzeichnis aufgelisteten 197 Veröffentlichungen Meyers ${ }^{39}$

\footnotetext{
${ }^{36}$ Zu Lise Meitner: Ruth Lewin SIME: Lise Meitner. Ein Leben für die Physik. Biographie. Frankfurt/Main 2001, u. Hannelore SEXL - Anne HARDY: Lise Meitner. Reinbek 2002. Über Meitners Zeit am Institut für Radiumforschung vgl. ebda, S. 32-38, sowie Meitners retrospektiver Beitrag Lise MEITNER: Looking back, in: Bulletin of the Atomic Scientists 20.11 (1964), S. 2-7, bes. S. 2f.

${ }^{37}$ Jeweils AÖAW, Institut für Radiumforschung, K. 21.

${ }^{38}$ AÖAW, Institut für Radiumforschung, K. 29.

${ }^{39}$ Werkverzeichnis bei REITER, Stefan Meyer (wie Anm. 25), S. 125-143.
} 

liegen
vor.

Durch Meyers Redaktionstätigkeit für die Boltzmann-Festschrift anläßlich dessen 60. Geburtstages im Jahre 1904, in der 117 Beiträge zum Abdruck kamen, liegen 94 Manuskripte international renommierter Wissenschaftler vor. ${ }^{41}$

Über die alltägliche wissenschaftliche Arbeit am Institut ist nur sehr wenig Material überliefert. Allein die Experimente über die Radiumemanation in bezug auf den menschlichen Körper in den Jahren von 1930 bis 1932, die einige interessante Einblicke in verschiedene Selbstversuche geben, sind gut dokumentiert. ${ }^{42}$ Weiters scheinen sich die Mitarbeiter des Instituts während eines größeren Teils ihrer Arbeitszeit auch mit der Erstellung von Eichungen und Messungen beschäftigt zu haben. ${ }^{43}$ Viele Privatleute, die hofften, in ihren Quellen Radioaktivität nachweisen zu können, um ein Heilbad zu errichten, wandten sich an das Institut, um von dort eine entsprechende Bestätigung zu erhalten. Aber auch diverse in- und ausländische staatliche Stellen suchten um Analyse von Bodenproben an; insgesamt können 225 Auftraggeber festgestellt werden. Die Unterlagen bzw. die Duplikate der Zertifikate für die Erstellung von Radiumstandards für die beteiligten Länder seit 1912/13 befinden sich ebenso unter diesen Unterlagen wie die Begutachtungen in Gerichtsverfahren und über die Wirksamkeit von unterschiedlichen

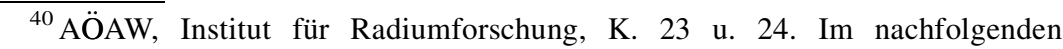
Archivbehelf ist zusätzlich die jeweilige Nummer des Werkverzeichnisses angegeben. Eine nahezu vollständige Sammlung von Sonderdrucken der Publikationen Stefan Meyers ist in der Österreichischen Zentralbibliothek für Physik (ZBPH) vorhanden. Sie befinden sich einerseits in zwei Aktenordnern, in denen Sonderdrucke mit unterschiedlichen Widmungen (z. B. an Haschek, Lecher oder Schweidler) aufbewahrt werden, und andererseits in einem Karton, in dem von einigen Publikationen sogar eine größere Menge von Sonderdrucken enthalten ist. Etliche dieser Sonderdrucke tragen die laufenden Nummern des von Meyer selbst angelegten Publikationsverzeichnisses und stammen sicherlich aus dessen Nachlaß. Der Inhalt des Kartons wurde der ZBPH im Jahre 1992 von Meyer-Biograph Wolfgang Reiter geschenkweise überlassen.

${ }^{41}$ AÖAW, Institut für Radiumforschung, K. 23-25. Seit der Darstellung von KARLIK, Briefwechsel (wie Anm. 27), S. 220, ist das Ms. von Louis Houllevigue (Beitrag-No. 9) in Verlust geraten.

${ }^{42}$ AÖAW, Institut für Radiumforschung, K. 29.

${ }^{43}$ AÖAW, Institut für Radiumforschung, K. 29-31. Otto HITTMAIR, Rutherford und das Wiener Radiuminstitut, (wie Anm. 31), S. 178, spricht sogar davon, das Radiuminstitut sei neben seiner Haupttätigkeit als Forschungsinstitut zum Eichamt für Radiumpräparate geworden. 
Emanationsapparaten, die ab ca. 1930 von Firmen für ein größeres Publikum entwickelt worden waren.

Für die Jahre von 1932 bis 1939 liegt Meyers Literaturkartei vor. In 25 Zigarrenkästchen befindet sich jahrgangsweise und innerhalb der Jahrgänge alphabetisch nach den Namen der Autoren geordnet eine Vielzahl von Karteikarten, auf denen Meyer die bibliographischen Angaben zu Fachpublikationen notierte. Ein Verzeichnis nennt die von ihm ausgewerteten Zeitschriften. ${ }^{44}$

In dem kleinen Konvolut „Humoristisches“45 befinden sich beispielsweise Exemplare der 10-Groschen-Nachtpost (ca. 1933) und des physikalischen Alphabets (s. d.), die wohl zu feierlichen Anlässen

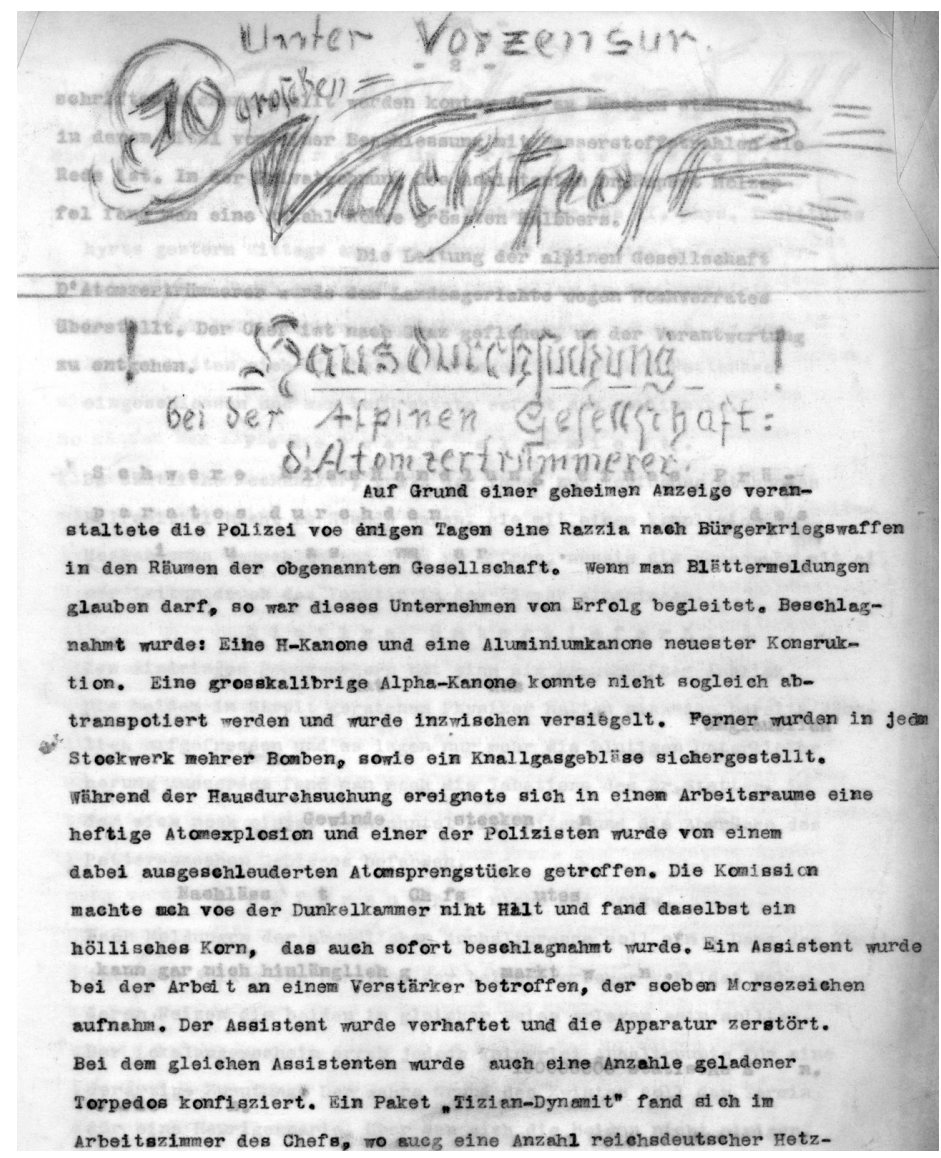

Abb. 4. 10-Groschen-Nachtpost (um 1933)

(AÖAW, Institut für Radiumforschung, K. 32) 
von Mitarbeitern des Instituts gedichtet und zusammengestellt wurden. So heißt es in der 10-Groschen-Nachtpost, S. 10, unter der Überschrift „Aus der okkulten Physik“:

Während einer Nachtsitzung im physikalischen Institute ist der als Medium bekannten Hertha Wambacher um 0:13 Uhr im Ultramikroskop das Negatropositelektroproton erschienen. Die Unglückliche wurde über diesen entsetzlichen Anblick ohnmächtig. Erst gegen Morgen konnte sie das Bewusstsein wieder erlangen, nachdem man ihr zwanzig Gramm Quecksilberverstärker eingeflösst hatte.

Auch in dem „Physikalischen Alphabet" wird darauf Bezug genommen: „Der Invar ist zu manchem gut, die Hertha schläft am Institut“. Auch der pseudo-wissenschaftliche Aufsatz „Über das natürliche Vorkommen des Elements 285 im Wiener Radiuminstitut" (ca. 1945) zeugt von dem Vergnügen, mit dem am Institut Forschung betrieben und mit einer gewissen Selbstironie beschmunzelt wurde. Sicherheitshalber notierte Berta Karlik viele Jahre später auf dem Manuskript „Scherzhafter Aufsatz“.

Der „X. Nachlaß Stefan Meyer" schließt mit den Kondolenzschreiben, die nach seinem Tod am Institut eingingen, sowie einigen dazugehörigen Dankschreiben Karliks. ${ }^{46}$

Die Akten „XI. Behördenschriftwechsel 1938-1944 (G. Ortner)“ (1/4 Karton) enthalten in erster Linie Anweisungen und Erlässe von offiziellen Stellen, wie der Universitätskanzlei und verschiedenen Reichsministerien, aber auch Korrespondenzen über die Anstellung von Erwin Fischer-Colbrie, der von der Universität Nürnberg nach Wien geholt wurde. Unter den Schriftstücken fällt eine Anfrage des Reichsministeriums für Wissenschaft, Erziehung und Volksbildung, Berlin, vom 29. Juli 1942 auf, in der Ortner um ,die Zusendung eines ausführlichen Gutachtens über die wissenschaftlichen Leistungen, das Lehrtalent und die persönliche Eignung “ von Rudolf Steinmaurer gebeten wurde, der für eine außerplanmäßige Professur vorgesehen sei. In seiner Antwort vom 26. August konzentriert Ortner sich allein auf die von ihm erwarteten Bereiche bezüglich Steinmaurers Qualifikation - die außerplanmäßige Professur am physikalischen Institut der Universität Innsbruck erhielt Steinmaurer im Jahre $1943 .{ }^{47}$ Von der Tätigkeit des Instituts in den Kriegsjahren zeugt schließlich

\footnotetext{
${ }^{44}$ AÖAW, Institut für Radiumforschung, K. 26-28; das Verzeichnis in K. 28.

${ }^{45}$ AÖAW, Institut für Radiumforschung, K. 32.

${ }^{46}$ AÖAW, Institut für Radiumforschung, K. 32.

${ }^{47}$ Vgl. Karl LINTNER: [Nachruf auf] Rudolf Steinmaurer, in: Almanach der ÖAW 150 (1999/2000), S. 407-417 (m. Abb.), hier: S. 413.
} 
noch ein Konvolut von 154 Briefen bezüglich der Anschaffung eines Neutronengenerators. Die Agende „Neutronengenerator“ ist nicht deutlich von der Tätigkeit des Vierjahresplaninstituts zu trennen, an das ab 1943 einige Schreiben in dieser Angelegenheit gerichtet sind.

\subsection{Nachlaß Karl Przibram}

Bei dem im AÖAW befindlichen Nachlaß des am 10. August 1973 verstorbenen Karl Przibram (,XII. Nachlaß Karl Przibram“, 7 Kartons), der auf dem Gebiet der Strahlenbeeinflussung fester Körper als „hervorragender Pionier“"48 angesehen werden kann, handelt es sich, ähnlich wie bei Stefan Meyer, in erster Linie um einen dienstlichen Nachlaß, der Einblicke in den beruflichen Werdegang und die wissenschaftliche Tätigkeit dieses Wiener Physikers erlaubt.

Einige Daten aus der wissenschaftlichen Laufbahn Przibrams spiegeln sich folglich in seinem Nachlaß wider. Nach dem Ersten Weltkrieg hatte Przibram im Jahr 1920 eine Assistentenstelle am Institut für Radiumforschung übernommen. 1927 ernannte man ihn zum außerordentlichen Professor an der Universität Wien. Dem Anschluß Österreichs an Hitler-Deutschland folgte seine Pensionierung. Die Zeit von 1940 bis 1946 verbrachte Przibram in der Emigration in Brüssel. 1946 wurde er von der Universität Wien als Ordinarius und Vorstand des II. Physikalischen Instituts zurückberufen.

Den zentralen Teil des Nachlasses bilden die Korrespondenzen, ${ }^{49}$ unter denen sich auch zahlreiche Schriftstücke privateren Charakters befinden. Gleichwohl erschien eine strenge Trennung zwischen privatem und dienstlichem Bereich aufgrund zahlreicher Überschneidungen weder sinnvoll noch möglich. Zwei Briefe aus der Korrespondenz mit Stefan Meyer, mit dem Przibram sowohl freundschaftlichen als auch wissenschaftlichen Kontakt pflegte, dienen dazu, um diese Verschränkung zu veranschaulichen: In seinem Brief

${ }^{48}$ Berta KARLIK, [Nachruf auf] Karl Przibram, in: Almanach der ÖAW 124 (1974), S. 379-387, hier: S. 379. Die folgenden Angaben gleichfalls hieraus. Vgl. auch Rudolf Werner SouKuP: Karl Przibram (1878-1973), Entdecker der Radiophotoluminiszenz, in: Rudolf Werner SouKuP (Hg.): Die wissenschaftliche Welt von gestern. Die Preisträger des Ignaz-L.-Lieben-Preises 1865-1937 und des RichardLieben-Preises 1912-1928. Ein Kapitel österreichischer Wissenschaftsgeschichte in Kurzbiografien. Wien-Köln-Weimar 2004 (= Beiträge zur Wissenschaftsgeschichte und Wissenschaftsforschung. 4), S. 263-266.

${ }^{49}$ AÖAW, Institut für Radiumforschung, K. 32-34. Vgl. hierzu Berta KARLIK: Register des wissenschaftlichen Briefwechsels von Karl Przibram, in: Sitzungsberichte der math.-nat. Klasse der ÖAW II 196 (1987), S. 49f. Ihr Beitrag ist jedoch bei weitem nicht vollständig und mit dem vorgelegten Archivbehelf überholt. 
vom 7. November 1945 bringt Meyer seine persönliche Freude zum Ausdruck, daß er wieder zu den Akademiesitzungen „einberufen“ wurde. Gleichzeitig setzt er Przibram davon in Kenntnis, daß er ihn als Vorstand des II. Physikalischen Instituts und Berta Karlik als Leiterin des Radium-Instituts vorgeschlagen habe. In einem Schreiben Przibrams an Meyer aus dem September 1947, dessen maschinenschriftlicher Durchschlag ebenfalls in Przibrams Nachlaß vorhanden ist, berichtet dieser von einem ,,sehr schönen Nachmittag bei Karliks in Mauer" und von einer Wurzelbehandlung seines Zahnes, infolge derer er eine ,geschwollene Backe“ gehabt habe, und sendet Meyer als Beilage eine Kopie einer elektronenmikroskopischen Aufnahme kolloider Goldteilchen. ${ }^{50}$

Die Korrespondenzen wurden für den Archivbehelf grundsätzlich nach dem Namen des Korrespondenzpartners und im Fall bestimmter Einrichtungen, wie beispielsweise bei Verlagen, Zeitschriften oder Universitätsinstituten, nach deren Sitz sortiert.

Mit dem Großteil der im Nachlaß überlieferten Partner korrespondierte Przibram im Zeitraum zwischen 1950 und 1970, wobei die meisten Konvolute durchschnittlich nicht mehr als ein bis fünf Schriftstücke enthalten. Unter den einzelnen Korrespondenzen machen die insgesamt 85 Briefe und Postkarten Stefan Meyers, die aus den Jahren von 1945 bis 1949 datieren, die Spitze aus; in größerem Abstand folgen 44 Schreiben Friedrich Paneths (19481957). Intensiven wissenschaftlichen Kontakt unterhielt Przibram kurzfristig wegen der englischen Übersetzung seiner 1953 erschienenen Monographie „Verfärbung und Lumineszenz“"51 mit dem Department of Physics der University of Durham, wovon 43 Briefe aus den Jahren von 1953 bis 1956 zeugen. Das Werk, das man international sehr anerkennend aufnahm, wurde - dies sei am Rand bemerkt - auch ins Russische übersetzt und wiederholt als Standardwerk bezeichnet. Ebenso korrespondierte Przibram mit Pergamon Press Ltd. (30 Briefe aus den Jahren von 1954 bis 1957) und mit der Redaktion der Zeitschrift ,Endeavour" in London in den Jahren 1953/54 (27 Briefe) in Hinblick auf die englische ebenso wie die spanische und italienische Fassung des Manuskriptes „Über die Bestrahlungsfarben in Mineralien“".52 Des weiteren haben sich aus

\footnotetext{
${ }^{50}$ Jeweils im AÖAW, Institut für Radiumforschung, K. 33.

${ }^{51}$ Karl PRZIBRAM: Verfärbung und Lumineszenz. Beiträge zur Mineralphysik. Wien 1953.

${ }^{52}$ Karl PRZIBRAM: Irradiation colours in minerals, in: Endeavour 13 (1954), S. 37-42.
} 
der Korrespondenz mit Herbert Steinmetz in München 34 Briefe erhalten (1951-1958).

Weiters sind $\mathrm{zu}$ nennen - trotz verständlicherweise geringeren Umfangs - Przibrams Schriftwechsel mit Berta Karlik mit 23 vorhandenen Stücken aus den Jahren von 1945 bis 1971 und derjenige mit seinem Schüler Franz Urbach, der später zum Leiter des Lumineszenzlaboratoriums der Firma Kodak in Rochester, New York, wurde, und dessen Frau Annie (28 Stücke, 1945-1958 und 1964).

Die frühesten Kontakte, die sich aus den im Nachlaß erhaltenen Einzelstücken ersehen lassen, datieren aus den Jahren 1911 (Brief Karl Spenglers aus Köln-Kalk) und 1921 (2 Briefe Maximilian Toeplers aus Dresden), sowie - um nur zwei Beispiele zu nennen von 1925 (Briefe der Universität Freiburg im Breisgau und Georg Hevesys aus Pest) und 1926 (Brief des Instituts für Edelsteinforschung in Idar-Oberstein). Ebenso haben sich in Hinblick auf Przibrams schriftliche Verbindung mit Victor Hess Einzelstücke aus den Jahren 1945, 1947, 1953 und 1960 erhalten. Als weitere prominente Beispiele seien die 15 Briefe und Karten Lise Meitners (1948-1966), zehn Schriftstücke Otto Hahns (1938-1966) sowie drei Sendungen Erwin Schrödingers (1946-1949) angeführt.

Mit Bezug auf Przibrams Kontakte zu Forschungseinrichtungen außerhalb Österreichs ist des weiteren festzuhalten, daß dem Nachlaß hier nur als Beispiele - Verbindungen zu dem „Kharagpur Indian Institute of Technology “ in den Jahren von 1965 bis 1967 (10 Briefe), zur University of Southern California in Los Angeles im Jahre 1947 (2 Briefe) und $\mathrm{zu}$ dem Lomonossoff Institut der Geochemie, Kristallographie und Mineralogie der Akademie der Wissenschaften in Moskau im Jahre 1935 (3 Briefe) zu entnehmen sind. In aller Regel wurden diese Schriftwechsel jedoch nur punktuell, einen spezifischen Anlaß betreffend, geführt.

In dem Nachlaß finden sich ferner zahlreiche Publikationen von Przibram selbst, ${ }^{53}$ die in renommierten Zeitschriften wie Nature, den Mitteilungen des Instituts für Radiumforschung oder der Zeitschrift für Physik veröffentlicht wurden und sich entsprechend Przibrams wissenschaftlichem Interesse den Fluoreszenzerscheinungen in verschiedenen Mineralien und den durch Bestrahlung hervorgerufenen Verfärbungen widmen. ${ }^{54}$ Auf dieser Ebene sind auch seine

\footnotetext{
${ }^{53}$ AÖAW, Institut für Radiumforschung, K. 34-37.

${ }^{54}$ Dazu auch KARLIK, Nachruf (wie Anm. 48), S. 382-384. AÖAW, Institut für Radiumforschung, K. 34-36.
} 
nachträglich angestellten Überlegungen und Verbesserungen $\mathrm{zu}$ seiner erwähnten Monographie „Verfärbung und Lumineszenz““ anzuführen. ${ }^{55}$

Die fremden Publikationen bezeugen vor allem Przibrams wissenschaftliche Kontakte mit Hans Adler, Engelbert Broda, Rudolf Steinmaurer, von denen sich jeweils mehrere Artikel in seinem Nachlaß erhalten haben. Auch von Bartholomew Nagy in New York sind drei Publikationen zu finden.

Überwiegend aus den 1940er und 1950er Jahren datieren die meist in Form maschinenschriftlicher Manuskripte vorhandenen Vorträge und Vorlesungen. ${ }^{56}$ Hieraus seien zwei am Institut für Wissenschaft und Kunst, Wien, in den Jahren 1946 und 1949 im Zusammenhang mit Formbildungen gehaltene Vorträge erwähnt, die nicht nur fachspezifischer Natur sind, sondern auch Przibrams philosophisches Interesse erahnen lassen. Anhand von Vergleichen aus dem praktischen Leben erklärte Przibram die Bedeutung der Morphologie, als einen Aspekt etwa die Kreisbildung in der organischen Natur, die

\section{RÉPULIQUE FRANĢAISE \\ UNIVERSITE DE PARIS \\ FACULTÉ DES SCIENCES \\ Année universitaire 1963-1964}

M. PRZIBRAM

Professeur à l'Université de Vienne

Invité dans le cadre des Echanges Culturels Franco-Autrichiens

fera deux conférences, dans l'Amphithéâtre de Chimie Physique, II, rue Pierre-Curie, Paris- $V^{\mathbf{e}}$, sur les sujets suivants :

$I^{\circ}$ LA fluorescence de traces organiques daNs les SUBSTANCES INORGANIQUES, le Jeudi 14 Novembre 1963, à $17 \mathrm{~h} 30$.

$2^{\circ}$ LA FLUORESCENCE DES FLUORINeS, le Vendredi 15 Novembre 1963 , à 15 h 30.

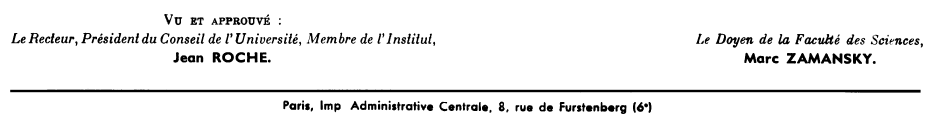

Abb. 5. Ankündigung zweier Vorträge von Karl Przibram (Plakat der Université de Paris) (AÖAW, Institut für Radiumforschung, K. 37)

\footnotetext{
${ }^{55}$ AÖAW, Institut für Radiumforschung, K. 35 u. 36.

${ }^{56}$ AÖAW, Institut für Radiumforschung, K. 37.
} 
sich leicht anhand der „ominösen kreisförmigen Schimmelkolonien auf schlecht eingekochter Marmelade" demonstrieren ließe. ${ }^{57}$ Auch versuchte er, den Unterschied zwischen den Formen im Organischen und im Anorganischen zu verdeutlichen, wofür er sich eines Zitates von Johann Wolfgang von Goethe bediente: „Denn keine Zeit und keine Macht zerstückelt/Geprägte Form, die lebend sich entwickelt" “58

Unter seinen wissenschaftlichen Arbeiten und Aufzeichnungen ${ }^{59}$ sind die Fachliteraturberichte Przibrams für die Union Minière du Haut Katanga zu nennen, für die er dank der guten Beziehungen zwischen dieser Gesellschaft und dem Radium-Institut in Wien während seiner Emigrationszeit in Brüssel arbeiten konnte. ${ }^{60}$ Das mit der Aufschrift „Rapatriement“ versehene eigene Konvolut dürfte von Interesse in Hinblick auf die Umstände sein, unter denen Przibram 1946 nach Wien zurückkehrte, weil hierin einige wenige Materialien zu diversen Korrespondenzen aus den Jahren 1945/46 enthalten sind. Da Przibram diesen Aktenkomplex selbst mit der genannten Bezeichnung versehen hatte, wurde er bei der Ordnung des Nachlasses als solcher belassen und als eigener Punkt unter den Korrespondenzen abgelegt. ${ }^{61}$

Für Przibrams Persönlichkeit und seine künstlerische Begabung ${ }^{62}$ sind des weiteren Gedichte und Erzählungen von Interesse, die auf privater Basis entstanden und auf seine „literarische“ Tätigkeit hindeuten. Es handelt sich dabei um einige Manuskripte, die er vermutlich in den Jahren 1971/72, also kurz vor seinem Tod, verfaßte und mit Titeln wie „Physiker-Träume“ oder „Begegnungen, die nie stattfanden " versah. ${ }^{63}$

Unter ersteren fällt etwa ein Traum des italienischen Arztes und Naturforschers Luigi Galvani auf, der für seine kränkliche Frau Froschschenkel besorgen und ihr für den Winter eine pelzgefütterte Pellerine kaufen sollte. Eines Nachts träumte er davon, daß seine Frau selbst grüne Froschschenkel hatte und ihre Frauenbeine neben seiner Elektrisiermaschine lagen, was mit unangenehmen Folgen verbunden

\footnotetext{
${ }^{57}$ Ebda, Konvolut IId, Manuskript u. d. T. „Formbildung in der anorganischen und organischen Natur; Versuch einer allgemeinen Morphologie“.

${ }^{58}$ Ebda, Konvolut IId, Manuskript u. d. T. „Formanalogien im Anorganischen und Organischen".

${ }^{59}$ AÖAW, Institut für Radiumforschung, K. 37.

${ }^{60}$ Vgl. KARLIK, Nachruf (wie Anm. 48), S. 385.

${ }^{61}$ AÖAW, Institut für Radiumforschung, K. 34.

${ }^{62}$ Hierzu auch KARLIK, Nachruf (wie Anm. 48), S. 386, die vor allem seine Scherenschnitte und Karikaturen hervorhebt.

${ }^{63}$ AÖAW, Institut für Radiumforschung, K. 37.
} 
war; doch lösen sich letztlich alle Probleme in Wohlgefallen auf. ${ }^{64}$ Hervorgehoben zu werden verdient ferner das Manuskript, in dem Przibram den Traum eines alten, längst emeritierten PhysikProfessors schildert, der sich, in einer Zeitung lesend, halb interessiert, halb angewidert über die raschen Veränderungen der Physik, über die Spezialisierung und die neuen Bezeichnungen ärgert, darüber einschläft und in eine Traumwelt versinkt, deren Schauplätze die Generalversammlung der UNO und seine alte Lehrstätte, die Universität, darstellen. ${ }^{65}$

Unter zweitgenannten findet man die Darstellung einer fiktiven Begegnung zwischen dem deutschen Physiker und Mathematiker Georg Christoph Lichtenberg (1742-1799) und Johann Wolfgang von Goethe (1749-1832) in Göttingen, die Przibram als, wie er es selbst bezeichnete, "Gedankenspielerei“" in schriftliche Form brachte: Nach einigen Fachsimpeleien zwischen den beiden Herren machte Lichtenberg eine spöttische Bemerkung über Goethes Irrtum in der Farbenlehre, worauf sich dieser ,ganz Exzellenz" kühl verabschiedete und Lichtenberg befürchten mußte, ,auf ein geistiges Hühnerauge getreten “ zu sein. ${ }^{66}$ Auch Johannes Kepler (1571-1630) und Galileo Galilei (1564-1642) weckten Przibrams Schreiblust, denn ,,in höherem Auftrag nach Pisa entsandt, steht Kepler in seinen Talar gehüllt, vor dem schiefen Turm und starrt hinauf" und trifft sogleich auf Galilei, der Kepler gesteht, daß ihm dessen elliptische Planetenbahnen „gar nicht gefallen“67. Erwähnt werden müssen des weiteren die "Stumpfsinn-Verse“, die Przibram in selbstironischem und melancholischem Licht in bezug auf sein hohes Alter erscheinen lassen: „Der Herr Professor / Weiss nichts besser. / Er sitzt in seinem Heime / Im Lehnstuhl drin, / sinnt vor sich hin / Und schreibt halt StumpfsinnReime. “68 Gern erinnerte Przibram sich an Boltzmanns Vorlesungen im „,baufälligen Hörsaal des alten physikalischen Instituts“ in der Türkenstraße 3, die dieser stets mit einem ,,sonoren ,Nun! “ “ begann, dessen hohe Stimmlage ,so gar nicht zu seiner wuchtigen Statur passte“", und die ,,von wunderbarer Klarheit“" waren. ${ }^{69}$

\footnotetext{
${ }^{64}$ Ebda, maschinenschriftliches Manuskript u. d. T. „Der Traum des Dr. Galvani““.

${ }^{65}$ Ebda, maschinenschriftliches Manuskript u. d. T. „,Der Traum eines Physikers des 20. Jahrhunderts".

${ }^{66}$ Ebda, maschinenschriftliches Manuskript u. d. T. „Lichtenberg und Goethe“.

${ }^{67}$ Ebda, maschinenschriftliches Manuskript u. d. T. „Kepler und Galilei““.

${ }^{68}$ Ebda, maschinenschriftliches Manuskript u. d. T. „Stumpfsinn-Verse““.

${ }^{69}$ Ebda, maschinenschriftliches Manuskript u. d. T. „Erinnerungen an Boltzmanns Vorlesungen“.
} 
Dem wissenschaftshistorisch interessierten Kenner werden seine Notizen zu diversen Experimenten und zu seinen eigenen Publikationen hilfreich sein. Eher spärlich sind hingegen die Aufzeichnungen aus dem Universitätsbereich, die Przibrams Funktion als Vorstand des II. physikalischen Instituts (1946-1951) belegen. ${ }^{70}$

Wendet man sich abschließend Przibrams öffentlichen Tätigkeiten in Gesellschaften, Vereinen oder auf Kongressen zu, so bietet der Nachlaß auch in dieser Hinsicht dem Bearbeiter Ansatz für verschiedene Fragestellungen, obgleich - verglichen mit den Nachlässen von Stefan Meyer oder Berta Karlik - deutlich weniger Material vorhanden ist. Hervorzuheben sind etwa seine Ämter als Obmann der Kommission für Strahlenforschung und Strahlenschutz von 1958 bis 1965 und des Kuratoriums des Instituts für Radiumforschung von 1950 bis $1965 .^{71} \mathrm{Zu}$ nennen sind weiters etliche Rundschreiben und Einladungen im Zusammenhang mit der Vereinigung Österreichischer Wissenschafter, der Przibram bis 1969 als Präsident vorstand, und seine Friedensaktivitäten beispielsweise im Österreichischen Hiroshima-Komitee. ${ }^{72}$ Als wirkliches Mitglied der Akademie (seit 1950) erreichten ihn freilich auch deren offizielle Zirkulare und Einladungen, doch enthält der Nachlaß zudem einige persönliche Mitteilungen von und an Przibram, wie etwa ein Schreiben des Präsidenten Erich Schmid aus dem Jahr $1964 .^{73}$

\subsection{Nachlaß Berta Karlik}

Der „XIII. Nachlaß Berta Karlik“ ist mit 26 Kartons der umfänglichste Teil der Akten des Instituts für Radiumforschung. Berta Karlik starb am 4. Februar 1990 im Alter von 86 Jahren als wirkliches Mitglied der Akademie. Nachdem sie ihre Studien der Physik und Mathematik im Jahr 1928 beendet und einen Auslandsaufenthalt in England verbracht hatte, wurde sie 1933 als wissenschaftliche Angestellte am Radium-Institut aufgenommen, dem sie nach dem Ausscheiden Stefan Meyers im Jahr 1947 bis zu ihrer Emeritierung 1974 vorstand. ${ }^{74}$

\footnotetext{
${ }^{70}$ AÖAW, Institut für Radiumforschung, K. 37 u. 38.

${ }^{71}$ AÖAW, Institut für Radiumforschung, K. 39.

${ }^{72}$ AÖAW, Institut für Radiumforschung, K. 38.

${ }^{73}$ AÖAW, Institut für Radiumforschung, K. 39.

${ }^{74}$ Karl LINTNER, [Nachruf auf] Berta Karlik, in: Almanach der ÖAW 140 (1990), S. 305-313, hier: S. 305f. Zuletzt: Art. „Karlik, Berta“ in: J. C. Poggendorff. Biographisch-Literarisches Handwörterbuch der exakten Naturwissenschaften. Band VIII/3 (2003), S. 1627-1629.
} 
Den umfangreichsten Teil ihres Nachlasses nehmen auch in diesem Fall die Korrespondenzen ein, die überwiegend aus den 1950er und 1960er Jahren datieren. ${ }^{75}$ Zwar ist im Vergleich zu Karl Przibram ungleich viel mehr Material überliefert, doch die Anzahl der erhaltenen Briefe und Karten, die Karlik mit einzelnen Personen oder Institutionen wechselte, ist auch hier durchschnittlich tendenziell gering, und häufig finden sich ebenfalls nur ein bis fünf Stücke pro Korrespondenzpartner.

Den weitaus größten Anteil machen die insgesamt 191 Briefe (1950-1970 und 1972-1987) vorwiegend des Präsidiums der ÖAW aus, bei denen es sich jedoch zumeist nur um allgemeine Rundschreiben handelt. Sodann sind 114 Briefe, Postkarten und Einladungen im Zusammenhang mit der Österreichischen Physikalischen Gesellschaft in Graz zu nennen, die den Zeitraum von 1951 bis 1979 umfassen. Innerhalb Österreichs sind des weiteren Karliks gut dokumentierte Verbindungen zur Universität Innsbruck anzuführen, darunter vor allem ihre 112 Briefe umfassende, aus den Jahren von 1949 bis 1973 datierende Korrespondenz mit Ferdinand Scheminzky, der zeitweilig auch am Forschungsinstitut in Bad Gastein tätig war. Besonders mit dem Elektrotechnischen Verein Österreichs in Wien korrespondierte Karlik über einen kürzeren Zeitraum hinweg sehr intensiv in Fragen der Atomenergie (64 Briefe von 1953 bis 1956 und von 1964); diese Unterlagen sind speziell auch im Zusammenhang mit dem Aufbau eines Szintillationsspektrometers für die Institutsgeschichte von Interesse. ${ }^{76}$

In regelmäßiger Verbindung stand Karlik auch zu dem Bundesministerium für Unterricht und verständlicherweise zum Dekanat der Philosophischen Fakultät der Universität Wien. Von Bedeutung für die Position und Geschichte des Wiener Radium-Instituts sind 81 Aktenstücke, die Einblicke in den Schriftwechsel der Isotopenstelle mit inner- und außerösterreichischen Partnern gewähren (1950-1967). Dem Bereich der beruflichen Kontakte ist desgleichen die äußerst dichte Korrespondenz zwischen dem Radium-Institut und etlichen anderen, vorwiegend universitären Einrichtungen in Österreich betreffend den Forschungsreaktor des Atominstituts der Universität Wien zuzurechnen.

\footnotetext{
${ }^{75}$ AÖAW, Institut für Radiumforschung, K. 39-57. Eine knappe Aufzählung ihrer Korrespondenzen legte Karlik noch zu Lebzeiten dar: Berta KARLIK: Register des Briefwechsels mit Wissenschaftlern, in: Sitzungsberichte der math.-nat. Klasse der Österreichischen Akademie der Wissenschaften II 195 (1986), S. 239-252.

${ }^{76}$ Siehe dazu oben S. 20.
} 
Umfangreiches Material hat sich im Zusammenhang mit dem Bundeskanzleramt für Auswärtige Angelegenheiten in Wien, speziell mit der „Beratenden Regierungskommission für die friedliche Anwendung der Atomenergie“, aus den Jahren von 1954 bis 1966 erhalten (91 Briefe). Auf Karliks dahin gehende Funktionen wird nachfolgend gesondert eingegangen.

Ein wenig ausführlicher sind in Summe betrachtet auch die Briefwechsel mit Hans Pettersson vom Oceanografiska Insitutet in Göteborg (73 Schreiben aus den Jahren von 1930 bis 1971), mit den Geschwistern Eva und Friedrich Adolf Paneth mit insgesamt 68 Sendungen aus dem Zeitraum 1958-1982 (Eva) und 1938 sowie 1946-1958 (Friedrich Adolf), mit Heinz Maier-Leibnitz von der Technischen Hochschule in München (65 Schreiben, 1952-1986), mit Josef Mattauch in Teilfingen und Mainz (60 Schreiben, 1944-1970), mit dem Bureau International des Poids et Mesures in Sèvres (54 Schreiben, 1960-1966 und 1985), mit Stefan Meyer in Bad Ischl (46 Schreiben, 1939-1949) sowie Walter Deisinger in Hanau am Main und New York (44 Schreiben, 1956-1968).

Weniger als 40 Stücke enthält der Briefverkehr mit Lise Meitner in Stockholm und Cambridge (36 Schreiben, 1938 und 1945-1963), mit Karl Przibram (34 Schreiben, 1939 und 1945-1973), mit Nikola Getoff (31 Schreiben, 1959-1973), mit Elisabeth Rona (32 Schreiben, 1939-1971), mit Otto Robert Frisch in Cambridge (30 Schreiben, 1948-1979) und mit den Philips Research Laboratories in Eindhoven (28 Schreiben, 1953-1968). Außerhalb jedes wissenschaftlichen Kontaktes stehen drei Briefe, die einen förmlich-freundschaftlichen Kontakt zu Kardinal Franz König andeuten, der Karlik im Jahr 1973 $\mathrm{zu}$ ihrer Wahl als wirkliches Akademiemitglied seine ,herzlichen Glückwünsche" "übersandte. ${ }^{77}$

Mit dem Österreichischen Gewerbeverein in Wien korrespondierte Karlik neben ihren ausschließlich wissenschaftlichen Aufgaben regelmäßig unter anderem in der Angelegenheit der Verleihung der Wilhelm-Exner-Medaille, die Karlik selbst im Jahre 1954 erhalten hatte. $^{78}$

Nicht uninteressant in Hinblick auf frauengeschichtliche Aspekte ist der Kontakt zwischen Karlik und Marietta Blau, wenngleich sich

\footnotetext{
${ }^{77}$ AÖAW, Institut für Radiumforschung, K. 43.

${ }^{78}$ Vgl. zur Wilhelm-Exner-Medaille: Herwig KAINZ (Red.): Symposium 75 Jahre Wilhelm-Exner-Medaille am Donnerstag, 7. November 1996, in der Österreichischen Akademie der Wissenschaften. Wien 1997.
} 
die erhaltene Korrespondenz eher spärlich ausmacht. ${ }^{79}$ Vor dem Zweiten Weltkrieg dürften sich die beiden Wissenschafterinnen recht nahe gestanden haben. ${ }^{80}$ Die beiden Briefe Blaus, die diese während eines wissenschaftlichen Aufenthalts in Göttingen 1932 als Antwort auf einen „lieben Brief“ an Karlik schrieb, lassen auf ein freundschaftliches Verhältnis schließen. Blau beklagte ein wenig das „,schrecklich bürokratische System“ in Göttingen, und daß ,für jeden Schmarrn [...] jemand anderer zuständig " sei. ${ }^{81}$ Nachdem ihr und ihrer Mitarbeiterin Hertha Wambacher noch im Jahr 1937 für ihre Untersuchungen der photographischen Wirkungen von Gammastrahlen der Ignaz-L.-Lieben-Preis der Akademie ${ }^{82}$ verliehen worden war, mußte Blau als Jüdin im darauffolgenden Jahr emigrieren. ${ }^{83}$ Als sie 1960 nach Österreich zurückkehrte, räumte man ihr eine „,bescheidene Möglichkeit“ ein, ihre wissenschaftlichen Arbeiten am Radium-Institut fortzusetzen. ${ }^{84}$ Karlik hatte davor Blau gegenüber bezeugt, daß sie sich ,riesig“ über deren Absicht freue, nach Wien zurückzukommen. ${ }^{85}$

Die Eindrücke über das Verhältnis zwischen Karlik und der nicht sehr gesprächigen, in persönlichen Dingen verschlossenen und generell zurückhaltenden Marietta Blau differieren. So hatte etwa Hannelore Sexl, deren Dissertation von Blau betreut wurde, nicht den Eindruck einer engen Freundschaft zwischen den beiden Frauen. ${ }^{86}$

\footnotetext{
${ }^{79}$ Insgesamt 14 Schriftstücke aus den Jahren 1932 bis 1970 (AÖAW, Institut für Radiumforschung, K. 39). Einige Briefe haben sich auch in der ZBPH erhalten, vgl. dazu Robert Rosner u. Brigitte STROHMAIER (Hg.): Marietta Blau. Sterne der Zertrümmerung. Biographie einer Wegbereiterin der modernen Teilchenphysik (=Beiträge zur Wissenschaftsgeschichte und Wissenschaftsforschung. 3), WienKöln-Weimar 2003, S. 87, Anm. 100 u. 101. Zu den wissenschaftlichen Verdiensten Blaus auch: FedERMAnN, Hess (wie Anm. 9), S. 78-81.

${ }^{80}$ Vgl. Rosner/Strohmaier, Marietta Blau (wie Anm. 79), S. 30.

${ }^{81}$ Brief Blaus an Karlik v. 22. Okt. 1932. AÖAW, Institut für Radiumforschung, K. 39.

${ }^{82} \mathrm{Zu}$ diesem Preis vgl. Robert Rosner: Der Ignaz Lieben Preis, in: Chemie. Das österreichische Magazin für Wirtschaft und Wissenschaft 4.4 (1997), S. 30. Der Ignaz-L.-Lieben-Preis wurde im Jahre 1937 mit der Verleihung an Blau und Wambacher zum vorläufig letzten Mal vergeben. Erst durch die Stiftung von Drs. Isabel und Alfred Bader konnte die OAW ab dem Jahre 2004 den Preis wieder vergeben. Vgl. hierzu SouKUP, Wissenschaftliche Welt (wie Anm. 48).

${ }^{83}$ Brigitte BISCHOF: Physikerinnen. 100 Jahre Frauenstudium an den Physikalischen Instituten der Universität Wien. Broschüre zur Ausstellung. Wien 1998, nicht paginiert, Artikel „Marietta Blau (1894-1970)“.

${ }^{84}$ Ebda.

${ }^{85}$ Brief Karliks an Blau v. 29. Juli 1958. AÖAW, Institut für Radiumforschung, K. 39.

${ }^{86}$ Rosner/STrohmaier, Marietta Blau (wie Anm. 79), S. 108.
} 
Gleichwohl erinnert sich Hanne Ellis-Lauda an eine bestehende Freundschaft, obgleich Karlik sich aufgrund ihrer zahlreichen Beschäftigungen nach Blaus Rückkehr nach Wien nicht sehr um sie gekümmert haben soll. ${ }^{87}$ In jedem Fall dürfte das Verhältnis nach Blaus Rückkehr „nicht frei von Spannungen“ gewesen sein. Zumindest zeigte sich Blau verletzt und enttäuscht über einige ihr zugetragene Bemerkungen der Institutsleiterin Karlik, die ihr nachsagte, sich kein Ansehen verschaffen zu können und sich ,,selber ins Eck" zu stellen. ${ }^{88}$ Nach Blaus Tod infolge eines schweren Lungenleidens kündigte Karlik einen Nachruf in den Acta Physica Austriaca an, der jedoch wegen einer schweren Erkrankung Karliks nie erschienen ist. ${ }^{89}$

Als prominentes Beispiel sei weiters der Briefverkehr mit Elisabeth Kara-Michailova in Sofia aus den Jahren von 1938 bis 1959 angeführt, wenngleich der Nachlaß auch hiervon nur sehr wenige Zeugnisse enthält.

Unter den außereuropäischen Verbindungen Karliks fällt auf, daß sie etwa in den Jahren 1960 und 1963 mit der High Voltage Engineering Corporation in Burlington/Massachusetts Schriftwechsel führte, des weiteren in den frühen 1950ern fallweise mit der University of Bandung in Indonesien betreffend eine Anstellung für Anny Frantz, zeitweise im Jahr 1954 mit John Irvine von dem Massachusetts Institute of Technology, 1955 mit der Transvaal and Delagoa Bay Investment Company Ltd. in Johannesburg und in den 1950er und 1960er Jahren mit verschiedenen wissenschaftlichen Instituten in Oak Ridge/Tennessee. Intensiveren Kontakt pflegte Karlik mit dem Argonne National Laboratory in Lemont/Illinois in den 1950er Jahren und im Jahr 1973. Etliche ihrer Verbindungen in die USA dürften durch ihre Studienreisen in den Jahren 1951 und 1956 zustande gekommen oder gefördert worden sein. Eine größere Anzahl von Unterlagen und Prospektmaterial zu diesen Reisen dokumentiert ein umfängliches Besichtigungsprogramm. ${ }^{90}$

In Anerkennung ihrer Qualifikationen wurde Karlik im Bundeskanzleramt für Auswärtige Angelegenheiten in eine Kommission zur Beratung in Fragen der Atomenergie berufen. ${ }^{91}$ Der daran interessierte

\footnotetext{
${ }^{87}$ Ebda, S. 91f. Immerhin hatte Karlik Blau nach deren Rückkehr die Benutzung ihrer Wohnung angeboten; ebda, S. 64.

${ }^{88}$ Ebda, S. $68 f$.

${ }^{89}$ Ebda, S. $72 \mathrm{f}$.

${ }^{90}$ AÖAW, Institut für Radiumforschung, K. 65.

${ }^{91}$ Vgl. LiNTNER, Nachruf (wie Anm. 74), S. 311.
} 


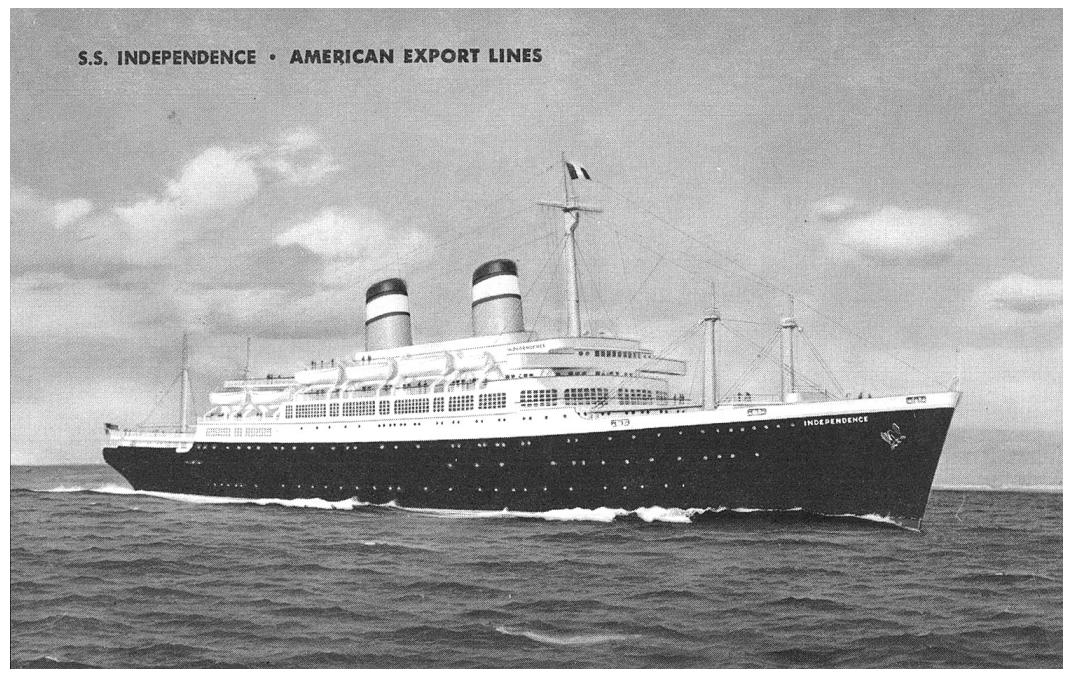

Abb. 6. Postkarte der S.S. Independence, mit der Berta Karlik ab dem 29. Aug. 1951 von Genua nach New York reiste (AÖAW, Institut für Radiumforschung, K. 65)

Benutzer findet zahlreiches Material aus den Jahren 1951 bis 1959 in ihrem Nachlaß. ${ }^{92}$ In einer Sitzung der österreichischen AtomenergieKommission wurde 1955 beschlossen, ein Memorandum auszuarbeiten, um festzuhalten, ,,in welcher Weise bisher Atomenergie in Österreich für friedliche Zwecke verwendet worden ist und welches gegenständliche Programm für die Zukunft geplant ist". Dabei mußte Karlik in Hinblick auf die Verwendung der Isotopen Wesentliches leisten. Ebenso war sie mit der Frage der Zweckmäßigkeit der Errichtung eines österreichischen Reaktors befaßt, für den nach ihrer Ansicht drei Anwendungsmöglichkeiten, namentlich die Erzeugung von Isotopen und von Energie ebenso wie Forschungszwecke, ins Auge zu fassen waren. ${ }^{93}$ Karlik war bemüht, auf die ihr durch das Kanzleramt regelmäßig zugesandten Resumées von den Sitzungen der genannten Kommission $\mathrm{zu}$ reagieren, indem sie einzelne Punkte klarzustellen versuchte - so betonte sie beispielsweise für das in Aussicht genommene Reaktorprojekt die Notwendigkeit der rein organisatorischen Vorarbeit und der Koordination von seiten der Wissenschaft. ${ }^{94}$

\footnotetext{
${ }^{92}$ AÖAW, Institut für Radiumforschung, K. 50.

${ }^{93}$ Beilage zum Schreiben des Bundeskanzleramtes für Auswärtige Angelegenheiten v. 27. Jan. 1955. Ebda.

${ }^{94}$ Brief Karliks v. 16. Mai 1955. Ebda.
} 
In diesem Zusammenhang sei zudem ihre Funktion als offizielle Vertreterin Österreichs an den beiden von der UNO in Genf in den Jahren 1955 und 1958 veranstalteten Konferenzen über die „Peaceful Uses of Atomic Energy " betont. Auf die dahin gehende Korrespondenz, der im übrigen zahlreiche Tätigkeits- und Sitzungsberichte und Memoranden beiliegen, wurde bereits kurz hingewiesen. ${ }^{95}$

Karlik erkannte die Wichtigkeit internationaler Zusammenarbeit auf dem Gebiet der Kernenergie und förderte - gegen anfängliche Widerstände - die Kooperation mit der Europäischen Organisation für Kernforschung (CERN). Besonders auch in Hinblick auf den Beitritt Österreichs bietet der Nachlaß verschiedene Materialien. Karlik hatte hierfür zahlreiche Referate vorzubereiten und mußte versuchen, eine einheitliche Stellungnahme zustande zu bringen, was sie nach ihren Aussagen ,viel Zeit und Mühe“ kostete. ${ }^{96}$ Diese Akten folgen, ähnlich wie bei Stefan Meyer, der von Karlik selbst angelegten thematischen Ordnung und wurden als ,thematische Korrespondenzen " abgelegt.

Der Beitritt Österreichs zum CERN erfolgte $1959,{ }^{97}$ und bereits für 1960 waren für junge österreichische Akademiker zehn Stipendien vorgesehen, um in Genf als technisch-wissenschaftliche Angestellte mitzuarbeiten. ${ }^{98}$ Bereits 1952 hatte Karlik für die Aufnahme Österreichs in den - gleichsam als Vorläufer des CERN - neu ins Leben gerufenen „Europäischen Forschungsrat für Kernphysik“ plädiert und auf den großen Nachteil hingewiesen, der Österreich erwachsen würde, ,wenn es an dieser ersten umfassenden europäischen wissenschaftlichen Arbeitsgemeinschaft nicht teilhätte", sowie betont, daß ,ein Fernestehen [‥] sein kulturelles Ansehen schwer beeinträchtigen“" würde. ${ }^{99}$

\footnotetext{
${ }^{95}$ Vgl. oben S. 41. Weitere diesbezügliche Stücke wurden in der Korrespondenz unter New York, UNO, eingeordnet. AOAW, Institut für Radiumforschung, K. 46; vgl. auch die Querverweise unter Wien, Bundeskanzleramt für Auswärtige Angelegenheiten sowie weiters unter den thematischen Korrespondenzen, Genf, CERN, (ebda, K. 56). Zudem LinTNER, Nachruf (wie Anm. 74), S. 311.

${ }^{96}$ Brief Karliks an Blau v. 29. Juli 1958. AÖAW, Institut für Radiumforschung, K. 39.

${ }^{97}$ Vgl. hierzu [Meinhard Regler]: Geschichte des österreichischen CERNBeitrittes in: 25 Jahre experimentelle Hochenergiephysik in Österreich. Mitgliedschaft bei CERN (Europäisches Laboratorium für Teilchenphysik). Hg. v. Institut für Hochenergiephysik der ÖAW. Kufstein-Wien-Heidenreichstein 1984, S. 11.

${ }^{98}$ Brief des Bundesministeriums für Unterricht an die Rektorate der wissenschaftl. Hochschulen und an das Dekanat der philosophischen Fakultät der Universität Wien v. 1. Sept. 1959. Ebda.

99 „Pour memoire“ Karliks v. 18. Okt. 1952. AÖAW, Institut für Radiumforschung, K. 55, Genf/CERN, Konv. 1.
} 
Nach dem Zweiten Weltkrieg setzte sich Karlik für den Rücktransport des in Salzburg ausgelagerten Radiums ein. ${ }^{100}$ Sie selbst gibt an, Ende September des Jahres 1945 durch einen ,glücklichen Zufall “ über die dortige Verwahrung größerer Radiummengen durch amerikanische Behörden erfahren zu haben. Diese hatten vermutet, daß das Radium im Zusammenhang mit einem Spital Anwendung gefunden hatte, jedoch konnte der Nachweis, daß es sich dabei um Präparate des Instituts handelte, rasch erbracht werden. Mitte Oktober reiste Karlik auf Aufforderung der amerikanischen Militärregierung nach Salzburg, wo der radioaktive Stoff in einem halbverfallenen, bewachten Bunker fern von jeglicher Menschenbehausung untergebracht war. Das Radium wurde schließlich in einem von den Amerikanern zur Verfügung gestellten Lastwagen, auf dem nach gewissenhaften Berechnungen eine fast einen Meter dicke Ziegelwand zum Schutz des Fahrers aufgebaut worden war, in Begleitung eines bewaffneten Jeeps wieder nach Wien gebracht. ${ }^{101}$ Im Verlauf des Winters gelangten dann auf amerikanische Kosten auch andere verlagerte Präparate zurück nach Wien. ${ }^{102}$ Über den Rücktransport des Radiums, das nun der Wissenschaft und der Medizin wieder ,als kostbarer Schatz" zur Verfügung stünde, über die Radiumforschung in Österreich, die Kooperation mit ausländischen Einrichtungen und den Wiederaufbau des Radium-Instituts berichtete auch die Zeitschrift ,Radio Wien“ im Mai 1946. Der Artikel zeigt sich bemüht, positive Aspekte der Radiumanwendung, wie etwa die Anwendung in Heilbädern, darzustellen, aber auch die Gefahren im Umgang mit radioaktivem Material aufzuzeigen. ${ }^{103}$

Einigen Aspekten von Karliks wissenschaftlichen Untersuchungen und Ambitionen läßt sich anhand der erhaltenen Notizbücher nachspüren, in die neben Experimenten, Meßergebnissen und Versuchsauswertungen auch ihre persönlichen Überlegungen, so beispielsweise zum Element 85, Eingang fanden. ${ }^{104}$

Besonders hervorzuheben ist die Lehrtätigkeit Karliks. ${ }^{105}$ Nach ihrer Habilitation im Jahr 1936 hielt sie ab dem Wintersemester

\footnotetext{
${ }^{100}$ Auch hierzu haben sich diverse Bescheinigungen und Listen der verlagerten Präparate in Karliks Nachlaß erhalten. AÖAW, Institut für Radiumforschung, K. 55. ${ }^{101}$ Vgl. KARLIK, 1938 bis 1950 (wie Anm. 5), S. 38f., u. LINTNER, Nachruf (wie Anm. 74), S. 311.

${ }^{102}$ Vgl. KARLIK, 1938 bis 1950 (wie Anm. 5), S. 39.

${ }^{103}$ AÖAW, Institut für Radiumforschung, K. 8, Zeitungsausschnitt „Wie das Radium nach Wien zurückkam“, in: Radio Wien, Heft 5 v. 18. Mai 1946.

${ }^{104}$ AÖAW, Institut für Radiumforschung, K. 62 u. 63.

${ }^{105}$ LINTNER, Nachruf (wie Anm. 74), S. 312.
} 
1937/38 regelmäßig Vorlesungen; etliche ihrer Vorlesungsmanuskripte haben sich im Nachlaß erhalten. Im Gegensatz zu vielen ihrer Kollegen, darunter auch Stefan Meyer, war Karlik von dem Anschluß an Hitler-Deutschland nicht direkt betroffen, und ihre akademische Laufbahn zeigt sich daher als relativ geradlinig. Nach dem Zweiten Weltkrieg wurde sie mit der provisorischen Leitung des RadiumInstituts betraut und übernahm somit die Verantwortung für den Wiederaufbau des Instituts. ${ }^{106}$ Im Jahr 1956 wurde sie zur ersten weiblichen Ordinaria der Universität Wien ernannt. Von der großen Anzahl ihrer Studenten zeugen die entsprechenden Aufzeichnungen aus den Jahren von 1945 bis 1974, bei denen es sich vornehmlich um Dissertationsgutachten handelt. ${ }^{107}$

Gleichsam als Vorbereitung ihrer Nachfolge datieren aus dem Jahr 1973 Stellungnahmen und Informationen über diverse, für das Ordinariat zur Diskussion stehende Kandidaten, mit denen Karlik auch persönlich korrespondierte. ${ }^{108}$

\subsection{Splitternachlässe und Glasplattennegative}

Die „XIV. Splitternachlässe“ (4 Kartons) enthalten Materialien, die als Provenienz verschiedener Wissenschaftler und Mitarbeiter des Instituts für Radiumforschung erkannt werden konnten: Traude Bernert, Ludwig Boltzmann, Felix Ehrenhaft, Franz Exner, Otto Hönigschmid, Gustav Jäger, Anton Lampa, Josef Loschmidt, Gustav Ortner, Fritz Paneth, Nikolaus Riehl, Elisabeth Rona, Erich Schmid, Egon von Schweidler, Hans Thirring und Carl Ulrich. Es handelt sich in aller Regel nur um einige wenige Briefe oder Postkarten oder um Sonderdrucke, die der entsprechenden Person gewidmet wurden. Einen etwas bemerkenswerteren Umfang hat der Nachlaß Franz Exner ${ }^{109}$ mit immerhin 34 Briefen und 23 Sonderdrucken; außerdem ist das Manuskript Exners zu dem Kapitel „Die Materie und ihre Konstitution“ "aus seinem Buch „Vorlesungen über die physikalischen Grundlagen der Naturwissenschaften" (Wien 1919) vorhanden und die Reinschrift von Exners Manuskript „Der schlichten Astronomia I. und II. Theil", wobei letzteres zusammen mit einem ..gedruckten Exemplar (Wien 1908) erst im Dezember 2002 der ÖAW durch

\footnotetext{
${ }^{106}$ Brigitte BISCHOF: Berta Karlik, in: Brigitta KeINTZEL u. Ilse KoROTIN (Hgg.): Wissenschaftlerinnen in und aus Österreich. Leben, Werk, Wirken. WienKöln-Weimar 2002, S. 353-356, hier: S. 354.

${ }^{107}$ AÖAW, Institut für Radiumforschung, K. 64.

${ }^{108}$ AÖAW, Institut für Radiumforschung, K. 57.

${ }^{109}$ AÖAW, Teilnachlaß Franz Seraphin Exner, 2 K.
} 
Exners Urenkel Peter C. Dijkgraaf, Den Helder, zum Geschenk gemacht wurde. Aus dem Besitz Stefan Meyers stammen die Un-Festschrift ,Der Jubel-Bandwurm. Vierteljahrhundert-Schrift zu Ehren Professor Franz Exners am 3. Februar 1904" und wahrscheinlich auch die Worte Benndorfs, die dieser am Grabe Exners am 18. November 1926 gesprochen hat (maschinenschriftliches Manuskript) sowie dessen Gedenkrede anläßlich der Enthüllung des ExnerDenkmals in der Universität Wien am 23. Januar 1937.

Der Splitternachlaß Fritz Paneth ist dominiert von 485 Briefen und Postkarten aus dem Schriftverkehr mit Georg von Hevesy, die allein in Fotokopie vorliegen und - vermutlich von Berta Karlik - aus dem Nachlaß Hevesys beschafft wurden. ${ }^{110}$ Von Paneth selbst stammen seine Vorlesungsmitschriften, worunter sich auch eine über „Experimentalphysik II (Wärme, Elektrizität)“ von Wilhelm Röntgen befindet, den er im Sommersemester 1908 an der Universität München hörte. Seine Mitschrift einer Vorlesung von Stefan Meyer (,Radioaktivität“, Universität Wien, Wintersemester 1911/12) ist umso interessanter, als sich in dessen Nachlaß keine Skripten zu eigenen Vorlesungen befinden. Zum Nachlaß Paneth gehört auch ein Brief Lawsons vom 29. Januar 1918 mit einigen beiliegenden Versuchsproben, die sich als einzige ,Materialien " in die ansonsten rein schriftlichen Unterlagen des Instituts geschlichen haben. Eine am 24. Juli 2001 durchgeführte Untersuchung hat ergeben, daß eine Probe nach wie vor radioaktiv strahlt und nicht mit bloßen Händen berührt werden sollte. ${ }^{111}$

Im Splitternachlaß Elisabeth Rona befinden sich 44 Briefe, fast ausschließlich aus den Jahren von 1934 bis 1937, darunter von Ellen Gleditsch, Georg Hevesy, Elisabeth Kara-Michailova, Agathe und Stefan Meyer sowie Hans Pettersson. ${ }^{112}$ Unter den wenigen Unterlagen im Splitternachlaß Egon von Schweidler befindet sich immerhin das Manuskript seiner Antrittsvorlesung, von dem jedoch nur noch Fotokopien und der knappe Vermerk vorliegen, daß das Original am 27. April 1978 von Berta Karlik an Dieter Flamm übergeben wurde. Weiters sind seine Aufzeichnungen über die Sitzung der Internationalen Radium-Standard-Kommission am 27. März 1912

${ }^{110}$ AÖAW, Institut für Radiumforschung, K. 65-67.

${ }^{111}$ AÖAW, Institut für Radiumforschung, K. 67.

${ }^{112}$ Ebda. Zu Elisabeth Rona vgl. REITER, Wissenschaftsemigration (wie Anm. 25), S. 718-720, und zuletzt Brigitte BISCHOF: Elisabeth Rona, in: KeINTZEL/ KoROTIN, Wissenschaftlerinnen (wie Anm. 106), S. 621-624 (m. weiteren Literaturhinweisen). 
in Paris vorhanden. ${ }^{113}$ Einer der interessantesten und zugleich unbekanntesten Männer im Umfeld der frühen Radium-Forschung ist Carl Ulrich (*1867 in Wien). Er hatte 1892 an der Universität Wien bei Adolf Lieben und Hugo Wiedl im Fach Chemie promoviert, ${ }^{114}$ und war zwischen 1910 und 1919 Direktor der k. k. Radiumfabrik in St. Joachimsthal. Als solcher saß Ulrich an einer der zentralen Stellen des internationalen Interesses an den Rohprodukten für die Radiumforschung. Aus seinem Nachlaß haben sich 95 Briefe erhalten, davon das Gros mit Stefan Meyer (45 Briefe), wozu sich die entsprechende Gegenkorrespondenz in dessen Nachlaß befindet. ${ }^{115}$

Die unzuordbaren Materialien (,XV. Varia“”, 1/4 Karton) konnten sehr gering gehalten werden. Es handelt sich beinahe ausschließlich um Unterlagen, die von Berta Karlik angelegt wurden bzw. aus der Zeit ihrer Leiterschaft des Instituts stammen. Sie wurden - soweit möglich - chronologisch sortiert.

Die „XVI. Fotos und Glasplattennegative“ (2 Kartons) bestehen aus Aufnahmen, die Stefan Meyer selbst gemacht oder gesammelt hat. Sie sind mit einer laufenden Nummer versehen worden und außerdem nach der Größe sortiert $(\mathrm{K}=$ klein, $\mathrm{M}=$ mittel und $\mathrm{G}=$ groß). Es liegen 101 kleine, 29 mittlere und 2 große Glasplattennegative bzw. -positive vor, wobei die kleinen in 76 private Aufnahmen Meyers (Reisen durch Italien und Österreich in den Jahren 1906-1908, u. a. mit Gustav Jäger) und 25 Aufnahmen mit Portraits von Physikern und von wissenschaftlichen Versuchen (1897-1915) unterschieden werden können (001-K bis $076-\mathrm{K}$ und $101-\mathrm{K}$ bis $125-\mathrm{K})$. Die mittelformatigen Glasplatten zeigen überwiegend ebenfalls Portraits von Wissenschaftlern (1888-1919) sowie einige „Platten für das periodische System und für die radioaktiven Substanzen für den Vortrag am 13. Febr. 1918 “ (301-M bis 329-M). Die beiden großformatigen Glasplatten zeigen Stefan Meyers von Radioaktivität belastete Hände (1910, 201-G u. 202-G).

${ }^{113}$ AÖAW, Institut für Radiumforschung, K. 68. Zur Person Schweidlers ist zu erwähnen, daß sich im AÖAW ein weiterer Teilnachlaß Schweidlers befindet, der neben einigen schriftlichen Aufzeichnungen, die überwiegend aus der Zeit seiner Tätigkeit als Mitglied des Präsidiums der Akademie der Wissenschaften stammen (Sekretär 1929-1933, Generalsekretär 1933-1938 und Vizepräsident 1938-1945), in erster Linie über 1.700 Sonderdrucke von befreundeten Wissenschaftlern enthält. Ein ausführliches Bestandsverzeichnis hierzu liegt im AÖAW vor und ist auch auf der Homepage des Archivs im pdf-Format einzusehen (http://www.oeaw.ac.at/biblio/ Archiv/Schweidler/schweidler.html).

${ }^{114}$ Archiv der Universität Wien, phil. Rigorosenakt Carl Ulrich, PN 722.

${ }^{115}$ AÖAW, Institut für Radiumforschung, K. 68 (Nachlaß Ulrich) bzw. K. 20 (Nachlaß Meyer). 


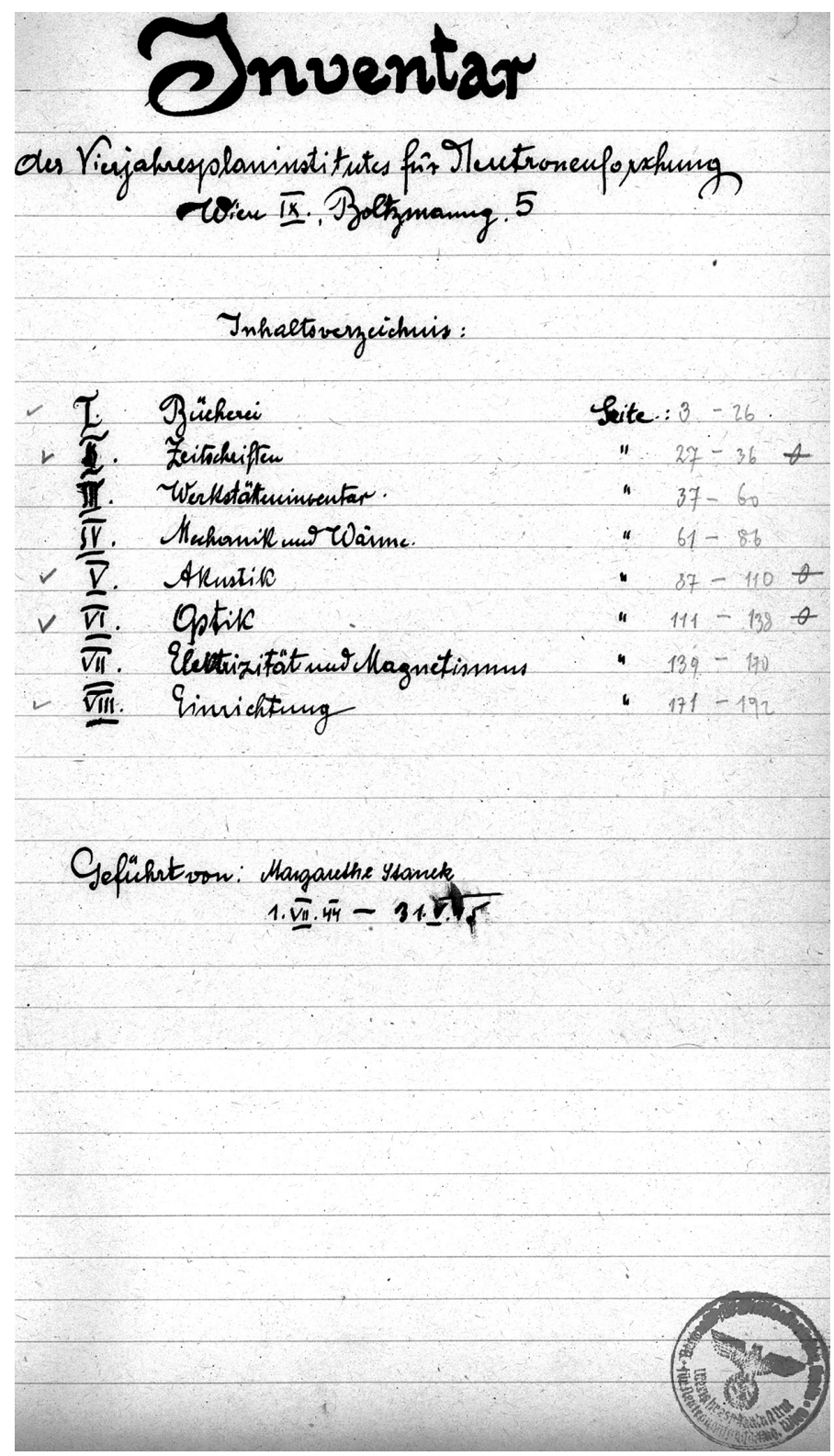

Abb. 7. Titelseite des Inventars des Vierjahresplaninstituts für Neutronenforschung (AÖAW, Vierjahresplaninstitut für Neutronenforschung, K. 1) 


\subsection{Vierjahresplaninstitut für Neutronenforschung}

Im Anhang des Archivbehelfs sind die Akten des „Vierjahresplaninstituts für Neutronenforschung " verzeichnet. ${ }^{116}$ Die Akten des Planinstituts wurden unter den Akten des Instituts für Radiumforschung gefunden, gehören aber nicht genuin in diese Provenienz, weswegen sie als eigenständiger Bestand bei einem Umfang von zwei Archivkartons geführt werden. ${ }^{117}$ Offenbar fanden die Akten aufgrund der Involvierung von Berta Karlik in die Liquidierung des Planinstituts Eingang in dieses Material.

Die Ordnung der Akten wurde augenscheinlich während der Liquidierung des Vierjahresplaninstituts für Neutronenforschung ab dem Sommer 1945 durch Rudolf Kraus angelegt - im Konv. 10 sind einige originale Aktenumschläge aufbewahrt. Gemäß einer Bestätigung des II. Physikalischen Instituts der Universität Wien vom 11. Okt. 1947 (Konv. 1) umfaßte das Aktenmaterial des liquidierten Vierjahresplaninstituts für Neutronenforschung folgende Akten: $\mathrm{A} / 1$, $\mathrm{A} / 2, \mathrm{~A} / 3, \mathrm{~A} / 4, \mathrm{~A} / 4 \mathrm{a}$ bis $\mathrm{A} / 4 \mathrm{k}, \mathrm{A} / 5, \mathrm{~A} / 6, \mathrm{~A} / 7, \mathrm{~A} / 8, \mathrm{~A} / 9$ u. $\mathrm{A} / 10$, von denen $\mathrm{A} / 1, \mathrm{~A} / 4 \mathrm{a}$ bis $\mathrm{A} / 4 \mathrm{f}, \mathrm{A} / 6$ u. A/8 zum Radium-Institut gekommen seien, während der Rest - mit Ausnahme der fehlenden Nummern A/3 u. A/4 - im II. Physikalischen Institut verwahrt werde. Die Ordnung wurde bei der Verzeichnung beibehalten, wobei die Aktennummer A/1 nicht zu verifizieren war. Möglicherweise handelt es sich um das Inventar des Vierjahresplaninstituts (Konv. 2). Außerhalb dieser Reihe stehen die Korrespondenzen Kraus' (April-Okt. 1945, Konv. 7) und die die Finanzen betreffenden Materialien (Konv. 8 u. 9).

Den Akten des Vierjahresplaninstituts für Neutronenforschung sind einige wenige Personalia des Vierjahresplaninstituts für Physikalische Chemie von Stoffgemischen (Leitung: Prof. Ludwig Ebert) aus Krausscher Provenienz beigegeben, der als Verwaltungsdirektor für beide Planinstitute verantwortlich war (Konv. 11).

\section{Zusammenfassung}

Die nun erschlossenen Archivalien eignen sich einerseits für fundierte Personenstudien, wofür im wesentlichen die im einzelnen sehr umfangreichen Nachlässe Stefan Meyers, Karl Przibrams und Berta Karliks zur Verfügung stehen. Diese enthalten zwar kaum persönliche

\footnotetext{
116 Über das Vierjahresplaninstitut für Neutronenforschung ist kaum mehr bekannt, als in Anm. 5 dargelegt werden konnte. Vgl. auch oben S. 33.

${ }^{117}$ AÖAW, Vierjahresplaninstitut für Neutronenforschung.
} 
Dokumente, doch lassen sich, nicht zuletzt aufgrund der in allen drei Fällen vielfältigen Korrespondenz, detaillierte Untersuchungen $\mathrm{zu}$ den jeweiligen Forschungsschwerpunkten, den freundschaftlichwissenschaftlichen Kontakten und in Einzelfällen - wie beispielsweise aus den privaten Aufzeichnungen Przibrams ersichtlich sogar zu persönlichen Neigungen anstellen. Der frauengeschichtlich orientierte Benutzer wird besonders den Nachlaß Karliks heranziehen, die im April 1945 mit der provisorischen Leitung des Instituts betraut und im Jahr 1973 als erste Frau zum wirklichen Mitglied der Akademie gewählt wurde. Generell ist dazu anzumerken, daß bereits unter der Ägide Stefan Meyers, der die Leitung des Instituts 1938 zurücklegen mußte, viele weibliche Mitarbeiter dort tätig waren. ${ }^{118}$

Interessiert man sich andererseits mehr für die Rahmenbedingungen des 1910 eröffneten Instituts für Radiumforschung, so finden sich auch dahin gehend zahlreiche Materialien betreffend den Status und die Verwaltung, die noch einer eingehenden Analyse harren. Dazu zählen etwa auch die Rechnungsbücher und zahlreiche Einzelbelege, die Aufschluß über die Finanzgebarung der Forschungseinrichtung geben. Auch liegen eigene Akten vor, anhand derer sich die Baugeschichte, die Erstausstattung und die hausinterne Buchhaltung beispielsweise in Hinblick auf die Anschaffungen bestimmter Gerätschaften - nachvollziehen lassen, um Rückschlüsse auf die Arbeitsbedingungen und wissenschaftlichen Errungenschaften ziehen $\mathrm{zu}$ können. In diesem Zusammenhang bieten auch die genannten Nachlässe zahlreiche Akten, die besonders Einblicke in internationale Kontakte und Kooperationen erlauben. Dabei ist auch an vermeintlich weniger bedeutsame Unterlagen zu denken, wie beispielsweise die Versandlisten der MIR. Zu Fragen der friedlichen Anwendung der Atomenergie nach dem Zweiten Weltkrieg offerieren einschlägige Akten im Zusammenhang mit dem CERN Antworten. Dieses Material entstammt dem Nachlaß von Berta Karlik, die als Mitglied der entsprechenden Kommissionen die Interessen des Instituts vertrat und dessen Stellenwert stets hervorzuheben wußte.

${ }^{118}$ Vgl. hierzu zuletzt Maria RENTETZI: Women in Physics. Women physicists in the Institute for Radium Research in Vienna, 1920-1938. A statistical report, in: Soziale Technik 11.2 (2001), S. 9-12. Ihre 2003 abgeschlossene Dissertation über „Gender, Politics, and Radioactivity Research in Vienna (1910-1938)“ wurde von der Virginia State University, Blackburg/VA, mit dem Outstanding Dissertation Award 2004 sowie von der American Historical Association mit dem Gutenberg e-prize ausgezeichnet. Hieraus zuletzt: Maria RENTETZI: Gender, Politics, and Radioactivity Research in Interwar Vienna, in: Iris 95 (2004), S. 359-393. 
Es läßt sich festhalten, daß auf Basis der vorhandenen Materialien, die der wissenschaftlichen Öffentlichkeit nun erstmals nach den Grundsätzen der modernen archivischen Verzeichnung geordnet zur Verfügung stehen, neben wissenschaftshistorischen Untersuchungen auch personengeschichtliche Studien durchzuführen sein werden.

Anschrift der Verfasser: Mag. Dr. Stefan Sienell, M.A.S., Archiv der Österreichischen Akademie der Wissenschaften, Dr.-Ignaz-Seipel-Platz 2, 1010 Wien, Österreich, E-Mail: stefan.sienell@oeaw.ac.at; Mag. Dr. Christine Ottner, M.A.S., Kommission für die Geschichte der Habsburgermonarchie der ÖAW, PrinzEugen-Straße 8, 1040 Wien, Österreich, E-Mail: christine.ottner@oeaw.ac.at. 